\section{A) Check for updates}

Cite this: Food Funct., 2020, 11, 6745

\title{
Dietary bioactive ingredients to modulate the gut microbiota-derived metabolite TMAO. New opportunities for functional food development
}

\author{
C. Simó (iD) and V. García-Cañas (D) *
}

There is a growing body of clinical evidence that supports a strong association between elevated circulating trimethylamine $\mathrm{N}$-oxide (TMAO) levels with increased risk of developing adverse cardiovascular outcomes such as atherosclerosis and thrombosis. TMAO is synthesized through a meta-organismal stepwise process that involves (i) the microbial production of TMA in the gut from dietary precursors and (ii) its subsequent oxidation to TMAO by flavin-containing monooxygenases in the liver. Choline, L-carnitine, betaine, and other TMA-containing compounds are the major dietary precursors of TMA. TMAO can also be absorbed directly from the gastrointestinal tract after the intake of TMAO-rich foods such as fish and shellfish. Thus, diet is an important factor as it provides the nutritional precursors to eventually produce TMAO. A number of studies have attempted to associate circulating TMAO levels with the consumption of diets rich in these foods. On the other hand, there is growing interest for the development of novel food ingredients that reduce either the TMAO-induced damage or the endogenous TMAO levels through the interference with microbiota and host metabolic processes involved in TMAO pathway. Such novel functional food ingredients would offer great opportunities to control circulating TMAO levels or its effects, and potentially contribute to decrease cardiovascular risk. In this review we summarize and discuss current data regarding the effects of TMA precursors-enriched foods or diets on circulating TMAO levels, and recent findings regarding the circulating TMAO-lowering effects of specific foods, food constituents and phytochemicals found in herbs, individually or in extracts, and their potential beneficial effect for

Received 12th May 2020 Accepted 10th July 2020 DOI: $10.1039 /$ dOfo01237h rsc.li/food-function cardiovascular health.

\section{Introduction}

There is mounting evidence that the metabolism of resident microbiota in the gut may have a crucial role in health and disease. $^{1,2}$ In recent years, there has been a growing interest in elucidating the interactions between gut microbiota metabolism and cardiovascular disease (CVD). Thus, it has become increasingly apparent that certain gut microbialderived metabolites, such as some bile acids, short-chain fatty acids, and trimethylamine- $N$-oxide, may contribute to CVD development and progression. ${ }^{3-11}$ The topic has attracted much attention as it can be deduced by the extraordinary number of review articles that can be found in a wide variety of scientific journals. Furthermore, new findings and discoveries in this area, such as the recent identification of the gut microbial metabolite phenylacetylglutamine and

Molecular Nutrition and Metabolism, Institute of Food Science Research (CIAL, CSIC-UAM), c/Nicolás Cabrera 9, 28049 Madrid, Spain.

E-mail: virginia.garcia@csic.es its implication in thrombosis potential and CVD, broaden our view of axis diet-microbiota-CVD. ${ }^{12}$ At the beginning of the 2010s, a series of groundbreaking studies associated elevated levels of circulating trimethylamine $N$-oxide (TMAO) with the development of atherosclerosis and increased risk of other adverse CVD events. ${ }^{13-15}$ Specifically, in a seminal work, Wang et al. (2011) uncovered a meta-organismal metabolic pathway that involves (i) the transformation of certain dietary trimethylamines (phosphatidylcholine, choline, betaine, L-carnitine, etc.) to trimethylamine (TMA) through gut microbiota metabolism, and (ii) its subsequent absorption in the intestine and delivery to the liver through the portal circulation, where it is oxidized to TMAO by flavin-containing monooxygenase family of enzymes (FMOs). ${ }^{13}$ Diet plays a key role in the production of TMAO since it provides with the nutritional precursors to produce TMA and TMAO. In addition, as it will be discussed in this review, other dietary constituents have also been investigated for their potential influence on endogenous TMAO levels or/and their effects against TMAO-induced damage, which would confer a dual role to diet for the prevention of CVD. 


\section{TMAO: from prognostic factor to therapeutic target}

There is a growing body of clinical evidence that supports a strong association between elevated plasma TMAO levels and cardiovascular risk as well as increased risk of adverse cardiovascular and mortality outcomes. The available clinical studies which investigate the role of TMAO in predicting prognostic outcomes, including mortality, in patients with several cardiovascular conditions have been reviewed. ${ }^{16,17}$ In addition to its prognostic value, accumulating data suggests that high plasma TMAO is a risk factor for CVD. A meta-analysis of prospective studies has shown that higher circulating levels of gut microbiota metabolites, including TMAO and its precursors, are associated with an increased risk of major adverse cardiovascular events, regardless of conventional risk factors. ${ }^{18}$ Various excellent reviews covering available studies on the association between circulating TMAO levels and CVD risk have been published in recent years and will not be addressed in the present work. ${ }^{17,19-22}$

In last years, the underlying molecular mechanisms by which TMAO promotes pathogenic effects on the cardiovascular system have attracted much attention. There are convincing evidences observed in vivo and in vitro studies suggesting that TMAO exerts a broad range of effects in diverse processes such as endothelial dysfunction, foam cell formation, thrombosis, and cholesterol metabolism, causing a variety of responses and outcomes, indicating that the effect of this metabolite is dependent on the cell type and the species. Human umbilical vein endothelial cells (HUVECs) has been one of the most studied in vitro models to explore the role of TMAO on the endothelial dysfunction, a hallmark of vascular damage that leads to atherosclerosis development. An early article by Sun et al. (2016) showed that TMAO stimulated the expression of nucleotide-binding domain, leucine-rich-containing family, pyrin domain-containing-3 (NLRP3) inflammasome via reactive oxygen species and thioredoxin-interactive protein. ${ }^{23}$ The immediate effect of such induction was the release of inflammatory cytokines interleukin (IL)-1B and IL-18. Further research using the same in vitro cell model supported these findings and concluded that inflammasome activation in part was mediated through inhibition of the SIRT3SOD2-mitochondial ROS signaling pathway. ${ }^{24}$ In a separate report, prolonged exposure of HUVECs to TMAO induced the expression of senescence markers and a reduction of cell proliferation among other effects that were suggestive of senescence and vascular aging. ${ }^{25}$ Additionally, Ma et al. (2017) observed that TMAO impaired endothelial self-repair and increased monocyte adhesion, processes that accelerate endothelial dysfunction, and subsequently, promote early pathological process of atherosclerosis. ${ }^{26}$ Interestingly, TMAO suppressed proliferation and migration of HUVECs compared to control cells, and at the molecular level, TMAO activated protein kinase $\mathrm{C}$ and $\mathrm{NF}-\mathrm{kB}$, and stimulated vascular cell adhesion molecule- 1 . The role of TMAO in the activation of inflammatory pathways in cells of the vasculature has also been investigated in vivo. A study on $\mathrm{LDLR}^{-1-}$ mice explored the impact of TMAO on smooth muscle and endothelial cell function. ${ }^{27}$ The elevation of inflammatory gene expression was a common observation in mice that were fed a choline diet or injected with an acute TMAO dose compared to control group. TMAO triggered mitogen-activated protein kinase, extracellular signal-related kinase, and NF-kB signaling cascade. Interestingly, the response observed at the molecular level was also reproduced in primary cultures of human endothelial and

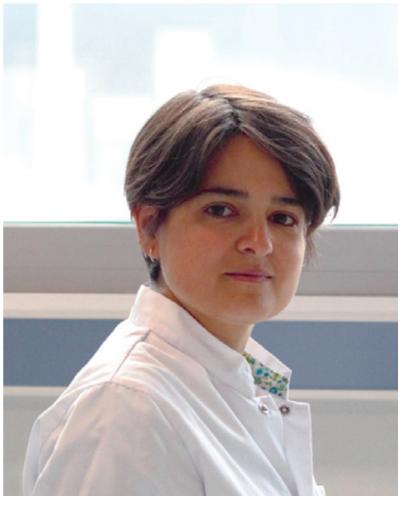

C. Simó
Carolina Simó, Ph.D., is Tenured Researcher at the Institute of Food Science Research (CIAL) that belongs to the National Research Council of Spain (CSIC). She obtained her doctorate degree in Chemistry in 2005. Her scientific career has involved 20 years of experience in the field of Food Science Research (https://orcid.org/00000002-7140-0795). She has specialized in the field of metabolomics applied to nutrition research to explore modulations of endogenous metabolic pathways in response to food intake and detect potential biomarkers of food consumption as well as the effects of a dietetic intervention. Simo's current research interests include the study new food ingredients with modulating activity of gut microbial metabolism related with disease development.

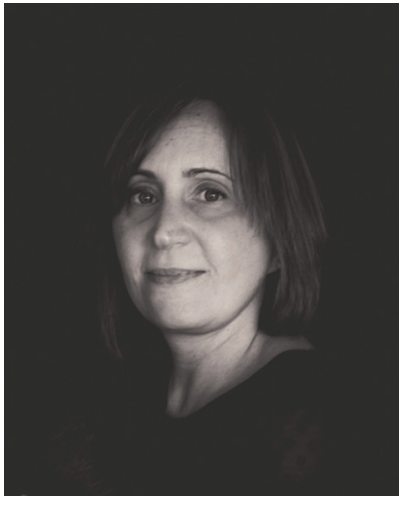

V. García-Cañas
Virginia Garcia-Cañas, Ph.D., Tenured Researcher at the Institute of Food Science Research that belongs to the National Research Council of Spain (CSIC). Specialized in omics technologies and bioactive ingredients (https://orcid.org/ 0000-0001-6728-4325).

Dr Garcia-Cañas research work focuses on the study of the biological activity and metabolism offoods and their constituents in different physiological and pathological conditions, and also, on the investigation of the underlying molecular mechanisms of bioactive food components. Her main research line is the study of the influence certain dietary constituents in the gut microbiota function and their impact in atherosclerosis and related diseases. 
smooth muscle cells, but it was not observed in mouse peritoneal macrophages subjected to similar treatments. Vascular calcification is a prevalent process in patients with chronic kidney disease (CKD). A recent study aimed at investigating the role of TMAO in vascular calcification revealed that TMAO induced gene expression of molecules involved in the osteogenic differentiation of human vascular smooth muscle cells. $^{28}$ In line with other studies, TMAO activated NLRP3 inflammasome and NF-kB signals during vascular calcification in CKD-induced rats, and inhibition of those signals diminished TMAO-induced calcification on vascular smooth muscle cells. Another recent article suggested that high-mobility group B1 (HMGB1) is one of the important mediators of TMAO-induced endothelial dysfunction and it is linked to Tolllike receptor 4 (TLR4) activation. ${ }^{29}$ It was found that EOMA endothelial cell monolayers exposed to TMAO exhibited reduced levels of various cell-cell junction proteins compared to untreated control cells, effects that were at least in part dependent of HMGB1 activation. Also related with HMGB1 activation, Cheng et al. (2019) have recently reported that exposures of HCAECs to a low TMAO concentration $(10 \mu \mathrm{M})$ promoted TNF- $\alpha$ or HMGB1-induced Tissue Factor (TF) expression. ${ }^{30} \mathrm{TF}$ is a molecule implicated in the thrombogenicity of atherosclerotic plaques and TMAO up-regulated its expression and activity in both concentration- and timefashion, changes that were also dependent on the activation of NF-kB.

Cholesterol accumulation in macrophages is a critical step in atherosclerosis. Wang et al. (2011) demonstrated that TMAO promoted uptake of cholesterol in macrophage by increasing the cell surface expression of two proatherogenic scavenger receptors, cluster of differentiation 36 (CD36), scavenger receptor $\mathrm{A}$ in macrophages. ${ }^{13}$ Further research showed that TMAO also induces the expression of ATP-binding cassette transporter A1 in macrophages which also favors the cholesterol uptake. ${ }^{14}$ Another relevant conclusion of the study in mice was that TMAO affects a major pathway for cholesterol elimination from the body. In fact, TMAO inhibited reverse cholesterol transport by affecting bile acid synthetic pathway in the liver at various levels, i.e. decreasing mRNA levels of bile acid synthetic enzymes Cyp7a1 and Cyp27a1, and multiple bile acid transporters (OATP1, OATP4, MRP2 and NTCP), observations that were not reproduced in the gut. Further, Geng et al. (2018) observed in vivo and in vitro that TMAO promoted CD36mediated transformation of ox-LDL induced macrophages into foam cells, a process that seemed to be mediated by MAPK/ JNK pathway in mice. ${ }^{31}$

With regard to the thrombogenic activity of TMAO various studies have been published. In a work by Zhu et al. (2016) plasma TMAO levels were independently correlated with incident thrombosis after a follow-up period of 3 years in a cohort with more than 4000 subjects. ${ }^{32}$ In vitro assays directly exposing platelets to TMAO elevated platelet hyperreactivity, with enhanced agonist-induced platelet activation through calcium release from intracellular stores. In addition, in vivo experiments in C57BL/6J mice confirmed the involvement of gut microbiota and TMAO in modulating platelet hyperresponsiveness. In a further report, Subramaniam et al. (2019) observed that cultured HAECs exposed to TMAO induced TF upregulation and procoagulant activity through NF-kB activation. ${ }^{33}$ However, contrasting results to those reported by $\mathrm{Zhu}$ et al. (2016) were found regarding the ability of TMAO to promote thrombosis in vivo. ${ }^{32}$

The aforementioned studies have provided valuable information about the molecular events that might explain biological effects on TMAO; however, the lack of understanding about the nature of the events that might trigger the molecular changes observed in the cells in response to TMAO has led to speculate on either two possibilities. On the one side, TMAO could be acting via its known effect on protein conformation and stability (i.e., as an allosteric modifier). On the other hand, TMAO could specifically interact with a receptor (i.e., as a ligand). This latter possibility seems to be more plausible considering the kinetics of TMAO-induced signaling responses in mammalian cells. In support of this notion, the recent report by Chen et al. (2019) has provided important insights and demonstrated that TMAO can directly and selectively interact with protein kinase R-like endoplasmic reticulum kinase (PERK), one of the three key molecular sensors in endoplasmic reticulum involved in the unfolded protein response signaling. ${ }^{34}$ Their results demonstrated in vivo and in vitro that TMAO-activated PERK induced FoxO1, a relevant transcription factor in metabolic disease; and the inhibition of TMAO formation in liver by FMO3 inhibitors or by manipulating mice gut microbiota could reduce PERK activation and FoxO1 levels and improve glucose tolerance. Considering the already published data supporting that PERK might induce activation of NLRP3 inflammasome, ${ }^{35} \mathrm{NF}^{\mathrm{kBB}},{ }^{36}$ and MAPK signaling, ${ }^{37}$ it can be anticipated that further research aimed at disentangling the potential links between these signaling pathways will help on uncovering the intriguing molecular mechanism underlying the pathogenic effects of TMAO in CVD.

\section{Key factors in TMA metabolism}

\subsection{Microbial metabolic pathways leading TMA production in gut}

The obligatory role for gut microbiota in TMAO formation from dietary trimethylamines has been demonstrated. Different gut microorganisms have different abilities to form TMA from dietary precursors. This is in part because there are various microbial pathways for its production in the gut (Fig. 1). Choline utilization (cut) gene cluster, responsible for anaerobic choline degradation, was shown to contain genes coding for the glycyl radical enzyme choline TMA-lyase (CutC) and its corresponding radical $S$-adenosyl-L-methionine (SAM) activating protein (CutD). ${ }^{38}$ Another microbial metabolic pathway generating TMA from L-carnitine is constituted by a two-component CntA/CntB oxygenase/reductase system capable of cleaving L-carnitine into TMA and malic semialdehyde. ${ }^{39}$ A closely related bacterial lyase consists of the yeaW/X 


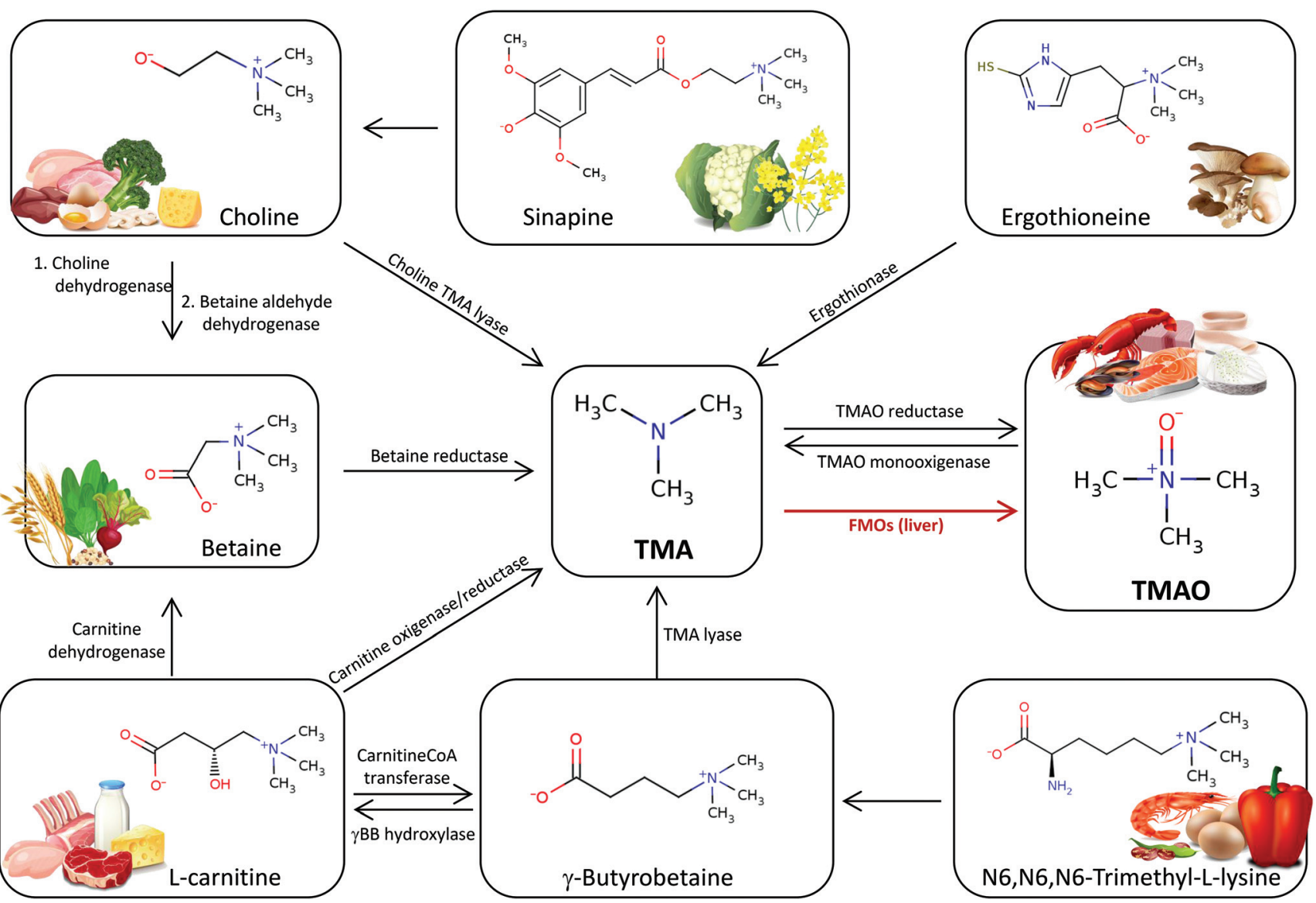

Fig. 1 Chemical formulas of main dietary precursors of TMA and TMAO. Schematic representation of the main metabolic pathways for the production of TMA by the gut microbiota and endogenous enzymes, and metabolism to TMAO by the hepatic FMOs.

gene products (YeaW/X TMA lyase). This promiscuous lyase can convert choline, betaine, L-carnitine, and $\gamma$-butyrobetaine to TMA. ${ }^{40,41}$ There are other enzymes that could be responsible for production of TMA from glycine betaine through the activity of glycine betaine reductase gene product ( $\mathrm{GrdH})$, an enzyme that requires selenium. ${ }^{42}$ Besides these TMA-generating pathways from dietary trimethylamines, an additional pathway that reduces TMAO to TMA by the activity of a torAlike gene product has been also described in some gut microorganisms. ${ }^{43}$ As will be discussed later, in addition to the mentioned phosphatidylcholine, choline and L-carnitine, other food nutrients possess a TMA moiety, suggesting that part of microbial TMA-generating pathways remain to be elucidated. Despite the relevance of TMAO to disease, the specific microorganisms responsible for the anaerobic dietary trimethylamines metabolism in the human gut remain unclear because the genetic and biochemical basis for utilization of these compounds is not fully characterized. Most of the reported attempts to correlate microbiota composition to plasma TMAO levels have been based on 16S ribosomal RNA gene (rDNA) amplicon sequencing technology. However, it has been demonstrated that such associations do not necessarily imply a causal link between the bacteria identified and the poten- tially enhanced production of TMA in the gut ecosystem. The relevant report by Romano et al. (2015) described eight strains, from two different phyla (Firmicutes and Proteobacteria) and obtained from human intestinal isolates, that were capable of producing TMA from choline in vitro. ${ }^{44}$ On the other hand, the application of advanced bioinformatics to screen reference genome libraries for species carrying the genetic potential to perform anaerobic dietary trimethylamines metabolism has proven to be a straightforward way of identifying potential gut TMA producers. The information derived from such screenings has been used for data mining in public genomic and metagenomic databases to provide a general overview of potential TMA-producing microorganisms in sequenced human gastrointestinal isolates. ${ }^{40,45}$ Using this strategy, TMA production potential has been detected in 102 reference genomes, covering 36 bacterial species. Although the TMA production function is widespread in Firmicutes, Proteobacteria, and Actinobacteria, it appears to be absent in Bacteroidetes. Remarkably, Clostridium spp., which were previously associated with an L-carnitine-rich diet using 16S rDNA amplicon sequencing, unexpectedly only pose CutC/D genes in their genome, which is associated with choline utilization, stressing the limitations of $16 \mathrm{~S}$ rDNA sequencing approach to identify 
novel organisms involved in TMA production. A bioinformatic study also suggested that TMAO reduction pathway has the highest detection rate in the gut metagenomes investigated, which indicates an intensive cycling between TMA and TMAO within the gut environment. ${ }^{45}$ Besides the interesting data provided by these novel bioinformatic approaches, it would be also important that findings about the metabolic potential of certain TMA producers could be validated biochemically.

\subsection{Dietary precursors of TMA and TMAO}

TMAO is found in a wide variety of fish and marine invertebrates since it plays an important role in their osmoregulation; it is involved in protein folded state stability and can counteract the effects of destabilizers such as urea and temperature. TMAO is also well known as indicator of freshness or postharvest spoilage degree of seafood products since, under refrigerated conditions, TMAO is reduced into TMA by specific spoilage organisms. ${ }^{46}$ TMA is the primary component that imparts the undesirable malodor that finally causes consumer rejection of poor-quality fish products. Marine fish and shellfish are the richest dietary source of TMAO. Concentrations in fresh seafood can vary extensively between species, habitat depth, season, etc. ${ }^{47}$ Significant differences in the TMAO concentrations between fish of various genera and species have been found ${ }^{48}$ with values ranging between 8 and $789 \mathrm{mg}$ per $100 \mathrm{~g}$ for ray-finned fishes and 262 and $789 \mathrm{mg}$ per $100 \mathrm{~g}$ for cartilaginous fishes.

L-Carnitine is considered a conditionally essential nutrient because, under certain conditions, it may require exogenous supplementation. L-Carnitine is essential for energy metabolism and its main function is the transport of long-chain fatty acids from the cytoplasm into mitochondria for $\beta$-oxidation. $\mathrm{L}$-Carnitine is endogenously synthesized from methionine and lysine in the liver, kidney, testis, heart, and brain. Besides its endogenous origin, L-carnitine can also be provided through the food intake; although, no dietary reference values have been set. L-Carnitine is generally found at high concentration in foods from animal origin (e.g. meat and dairy products), and in lesser amounts can be found in grains and vegetables. ${ }^{49}$ Red meat is the richest dietary source of L-carnitine; it has been reported that the content of L-carnitine in beef range from 45 to $66 \mathrm{mg}$ per $100 \mathrm{~g}$, although higher amounts have been found in the muscle from kangaroo and horse. ${ }^{50}$ L-Carnitine concentrations in dairy products vary widely with the different products, consistently with fat content and animal origin, ranged from 3 to $42 \mathrm{mg}$ per $100 \mathrm{~g}$ in a wide range of dairy products such as milk, yogurt, cheese and cream. ${ }^{50}$

Choline is also considered a conditionally essential nutrient for humans since the amount produced endogenously in the liver is not sufficient to meet human requirements, and thus, it needs to be obtained from dietary and supplement sources. The Panel on Dietetics Products, Nutrition, and Allergies from the European Food Safety Authority (EFSA) set the adequate intake (AI) at $400 \mathrm{mg} \mathrm{day}{ }^{-1}$ for men and women, ${ }^{51}$ while the American Institute of Medicine (IOM) set a different AI for men $\left(550 \mathrm{mg}\right.$ day $\left.^{-1}\right)$ and women $\left.\left(425 \mathrm{mg} \mathrm{day}^{-1}\right)\right)^{52}$ The main forms of choline present in foods are both water-soluble: free choline, phosphocholine, (PChol), glycerophosphocholine (GPC), and lipid-soluble: phosphatidylcholine (PC) and sphingomyelin (SPM). Other minor amounts are the water-soluble forms cytidine-5-diphosphate-choline (CDP-choline) and acetylcholine. Animal-derived foods contain more choline than plant-based ingredients, but certain plant-based foods are also rich in choline and choline-containing molecules. Eggs and liver represent the major sources of choline in diets, followed by meats and fish, whole grain, cereal, vegetables, fruits, milk, fat and oils. ${ }^{53}$ Choline also exists in considerable amounts in the form of phenolic choline esters in the seeds of the Brassicaceae family. ${ }^{54}$ Sinapine (the choline ester of sinapic acid) is the best-known compound of this class due to its occurrence in foods such as cauliflower, and it has been studied as a dietary precursor of TMAO in rapeseed-derived feed for pigs. ${ }^{55}$ Besides sinapine a number of choline related compounds derived from substituted benzoic and cinnamic acids have been identified, however, their role as dietary precursors of TMA has yet to be thoroughly researched.

$N 6, N 6, N 6$-Trimethyl---lysine (TML) is a non-protein amino acid which has an important role as a precursor of $\mathrm{L}$-carnitine. It has been determined that TML is presented as free amino acid in noticeable amounts in vegetables, which represent an important part of daily alimentation for most mammals. Servillo et al. (2014) have reported the occurrence of TML in a number of vegetables sources such as legumes, grains, leafy vegetables, solanaceae vegetables and fruits. ${ }^{56}$ The highest concentration in vegetables was found in sweet pepper fruit (0.8-1.8 mg per $100 \mathrm{~g})$. Li et al. (2018) reported the presence of TML in both plant- and animal-derived foods alike, and they found a greater level in animal- than plant-derived products. ${ }^{57}$ The TML content in meats from different animal origins ranged from 1 to $8.6 \mathrm{mg}$ per $100 \mathrm{~g}$, and interestingly, amount TML was the highest in eggs ( $13.7 \mathrm{mg}$ per $100 \mathrm{~g}$ ) and shrimps (12.2 mg per $100 \mathrm{~g}$ ).

Betaine, a choline derivative also known as glycine betaine and trimethylglycine, is produced endogenously from choline and the amino acid glycine. Betaine is present in plant- and animal-based foods. Cereal-based foods are one of the major sources of betaine ( $>1 \mathrm{~g}$ per $100 \mathrm{~g}){ }^{58}$ Spinach, beets, crustaceans and finfish are also good sources of betaine $(650-200 \mathrm{mg}$ per $100 \mathrm{~g}) .^{59}$

Ergothioneine is an unusual sulfur-containing derivative of histidine that is synthesized only by some fungi and bacteria. In humans, ergothioneine is acquired exclusively through dietary sources. Only some food contain ergothioneine, with highest concentrations detected in boletus and oyster mushrooms (11.9-52.8 $\mathrm{mg}$ per $100 \mathrm{~g}$ ), and, to a lesser extent, in chicken and pork liver $\left(0.9-1.1 \mathrm{mg} \mathrm{mL}^{-1}\right)$, pork kidney $(0.8 \mathrm{mg}$ per $100 \mathrm{~g}$ ), black and red beans (0.4-1.3 $\mathrm{mg}$ per $100 \mathrm{~g})$, and oat bran $(0.4 \mathrm{mg}$ per $100 \mathrm{~g}) .^{60}$

Recently, $\boldsymbol{\delta}$-Valerobetaine has been described as a constitutive metabolite of ruminant meat and milk. The presence of $\delta$-valerobetaine has been reported in milk $\left(30-60 \mu \mathrm{mol} \mathrm{L}^{-1}\right)$ 


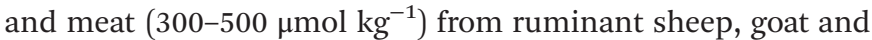
cattle. ${ }^{61}$ The levels of $\delta$-valerobetaine were considerably higher in ruminant (sheep, goat and cattle) than in non-ruminant (horse, rabbit, chicken and pig) meat. $\delta$-Valerobetaine was originated by the rumen microbiota from TML occurring in vegetable-derived ruminant diet. Moreover, the possible fate of $\delta$-valerobetaine expected from dietary source by humans was assessed, and it was observed that increasing production of TMA over time was obtained when $\delta$-valerobetaine was incubated with caecum content of a non-ruminat animal (rabbit) under anaerobic conditions.

Besides the aforementioned dietary precursors of TMA/ TMAO (Fig. 1), active research is underway to evaluate and discover other novel dietary precursors and the role of gut microbiota to release TMA from these substrates.

\section{The complex link between diet and endogenous TMAO levels}

\subsection{Association of TMAO levels with consumption of foods}

Studies on the association of urine or plasma TMAO levels with consumption of foods of animal origin, rich in dietary trimethylamines have demonstrated that TMAO production shows wide inter- and intra-individual variations. Circulating TMAO is determined by a number of factors including type of diet, liver FMO activity and kidney function. Genetic factors appear to play only a marginal role in plasma TMAO levels suggesting that interrelationships between dietary precursors and the gut microbes are more likely determinants of variation in plasma TMAO levels in healthy population. ${ }^{62}$

TMAO is present in significant quantities in marine fish and shellfish and represent a significant source of dietary TMAO for humans. Consistent results across different nutritional intervention and observational studies have shown that TMAO in urine and/or plasma is a strong indicator of dietary exposure to fish. The intake of fish has been mentioned as a cause of higher urine levels of TMAO in some observational studies. For instance, after metabolomic analysis of urine obtained from different population samples (852 participants from China, Japan and USA), it was noted that high levels of TMAO were particularly dominant in the Japanese population, consistent with the high dietary intake of fish in this area. ${ }^{63}$ Similarly, from cross-sectional data from the INTERMAP study, including 4680 participants from Japan, China, United Kingdom, and USA, urinary TMAO showed a stronger association with fish intake in the Japanese population sample. ${ }^{64}$ In the cross-sectional KarMeN study in 2012/2013, Krüger et al. (2017) also observed that fish intake was associated with increased TMAO concentrations in both plasma and urine samples from German adult population (297 participants). ${ }^{65}$

Several nutritional intervention studies have reported high levels of TMAO in urine and plasma after consumption of fish. Consumption of single meal with salmon was followed by substantial increased postprandial urinary concentrations of TMAO, ${ }^{66}$ suggesting that free TMAO present in fish can be readily absorbed without the involvement of gut microbiota. Likewise, longer-term intake of fish has also been associated with higher TMAO levels. For instance, $150 \mathrm{~g}$ seafood a day (salmon, cod, herring or shrimps) over a one-week period affected plasma and urine TMAO levels, which increased 16-fold and 6-fold, respectively, after the seafood-rich diet. ${ }^{67}$

When compared with other non-fish diets, it has been shown that TMAO is more closely associated with fish intake. ${ }^{68,69}$ In crossover meal studies, fish-rich diets have been associated with significantly higher plasma TMAO levels than meat- and egg-rich diets. Thus, a major increase in TMAO concentration in urine was observed after fish intake but not after meat (chicken, red meat and processed meat) intake over 3 weeks; ${ }^{68,70}$ also, urinary TMAO displayed a strong doseresponse relationship with fish intake. ${ }^{70}$ In a randomized, controlled crossover design with four arms comparing the effect of four different meals (fish, eggs, beef, and fruit control) taken in a single day, it was observed that fish consumption yielded $\sim 50$ times higher postprandial plasma concentrations of TMAO compared to eggs or beef intake. ${ }^{69}$

Unlike seafood, animal products do not contain free TMAO. Nevertheless, as mentioned above, there are a variety of TMAcontaining nutrients, primarily found in foods of animal origin, and to a lesser extent in vegetable products, that can be potentially dietary precursors of TMAO.

As previously mentioned, eggs are one of the major sources of choline (and its esterified forms) in diets. One egg contains $250 \mathrm{mg}$ of choline moiety per $100 \mathrm{~g}$ and one egg yolk contains $680 \mathrm{mg}$ of choline moiety per $100 \mathrm{~g} .{ }^{53}$ Eggs are also a good source of cholesterol, hereby raising the debate about associations among egg consumption and risk of CVD. It has been shown that acute intake of choline from eggs may influence postprandial plasma TMAO levels in healthy subjects. Highlighting the role of gut microflora in this process, Tang et al. (2013) showed that subjects who consumed a dietary PC challenge (hard-boiled eggs and deuterium (d9)-labeled PC) had acute increases in plasma TMAO in a gut microfloradependent manner. ${ }^{71}$ Miller et al. (2014) also observed a dosedependent increased plasma and urine TMAO concentrations at $24 \mathrm{~h}$ after a single meal exposure of 0, 1, 2, 4 or 6 egg yolks, with significant increases in TMAO concentrations from $\geq 2$ egg yolks, and the highest TMAO concentrations achieved at 6 and $8 \mathrm{~h}$ after the ingestion of the eggs. ${ }^{72}$

However, when egg intake trials were conducted for longer periods of time, differing results were obtained. For instance, West et al. (2014) observed that regular egg consumption (8-week period, 6 eggs per week) did not increased post-intervention fasted plasma concentrations of TMAO (compared with an egg-free control diet) in lacto-ovo-vegetarian women. ${ }^{73}$ These results agreed with data from the study reported by DiMarco et al. (2017), in which the intake of 1, 2, or 3 eggs per day for 4 weeks $(n=36)$ increased in a dose-dependent manner plasma choline concentrations while plasma TMAO remained unchanged for the duration of the dietary intervention. ${ }^{74}$ In subsequent crossover dietary interventions carried out by the same research group, the effect of 3 eggs per day intake $v s$. 
daily choline bitartrate supplementation $(n=30)^{75}$ and consumption of 2 eggs per day $v s$. oatmeal for breakfast $(n=30),{ }^{76}$ were evaluated on plasma choline and TMAO levels. The obtained results confirmed that after a 4-week intervention period, choline was significantly increased in plasma when administered in the form of eggs or as dietary supplement; however, neither egg intake nor choline supplement increased fasting plasma TMAO levels when compared to baseline values. In an observational study, Obeid et al. (2017) examined the type of the vegetarian diet and it was reported that vegans and lacto-ovo vegetarians that followed stable dietary patterns for a mean of 10 years did not differ in plasma concentrations of TMAO. ${ }^{77}$ Collectively, these results indicate that although acute egg ingestion increases postprandial plasma TMAO to some extent, there is not a clear association between egg consumption and increased fasting plasma levels of TMAO. Moreover, these works lack of gut microbiome information and the link between the microbiome and TMAO was not addressed, and thus, it should be further investigated.

It has also been found that depending on the structure of choline in different dietary sources and the foodstuffs in which they are present, absorption and metabolism may differ and impact the bioavailability of choline. This was exemplified by Mödinger et al. (2019) that compared the plasma kinetics of choline and its turnover into choline derivatives upon intake of two different choline sources: phosphatidylcholine from commercial krill oil and capsules containing choline bitartrate salt. ${ }^{78}$ In this regard, higher TMAO concentrations were measured after intake of the choline bitartrate, compared to phosphatidylcholine from krill oil. Interestingly, Berge et al. (2015) also observed that dietary supplementation with krill oil resulted in increased choline and betaine but not plasma TMAO levels. ${ }^{79}$

Meat constitutes a significant part of human diet since it is an important source of high-quality proteins and important micronutrients. ${ }^{80,81}$ Certain types of meats are also rich in fats and cholesterol as well as in L-carnitine and choline. ${ }^{50,53} \mathrm{~A}$ number of observational studies have investigated the effects of long-term dietary habits (e.g., vegan/vegetarian vs. omnivore) on the generation of TMAO. Several studies observed that baseline plasma TMAO levels were lower among vegan and vegetarian subjects compared to omnivores. ${ }^{14,82}$ In addition, urinary TMAO was found to be one of the most significant metabolites responsible for the differences observed between subjects following long-term (more than 5 years) lacto-vegetarian or omnivorous diets. ${ }^{83}$ In another report, TMAO generation was also significantly lower in vegans/vegetarians after 2-3 months of continuous daily supplemental L-carnitine ingestion. ${ }^{84}$ It has also been reported that both vegan/vegetarian and omnivore alike showed increases larger than 10 -fold in plasma TMAO levels after 1-2-month periods of choline supplementation. ${ }^{82}$ In another cross-sectional study by Kruger et al. (2017) it was reported that red meat but not white meat intake was positively associated with plasma TMAO concentrations. ${ }^{65}$

Human dietary interventional studies, aimed at discovering nutrition markers in body fluids, have found an association between red meat consumption and increased plasma or urine levels of TMAO. A short-term (15 days) randomized crossover study showed that high red meat diet $\left(420 \mathrm{~g} \mathrm{day}^{-1}\right)$ increased urinary TMAO when compared to vegetarian diets; however, no difference in TMAO was observed between a low meat diet $\left(60 \mathrm{~g} \mathrm{day}^{-1}\right)$ and vegetarian diet. ${ }^{85}$ In an intervention nutritional trial focused on various defined doses of meat (either chicken or beef) following a crossover design, TMAO was the only analyte that increased after beef intake (but not after chicken intake) and remained above baseline even after $24 \mathrm{~h} .{ }^{86}$ Moreover, after the ingestion of 0,100 , or $200 \mathrm{~g}$ beef, clear dose-response was observed for TMAO levels, with maximum plasma concentrations around $11 \mathrm{~h}$ after the intake. Similarly, in a randomized controlled crossover trial, the effect of 4 -weeks intake of red meat, poultry, and non-meat protein sources as $25 \%$ of their daily calories, was investigated, ${ }^{87}$ and it was observed that compared to the white meat or non-meat diets, increased plasma and urine levels of TMAO occurred after consumption of the red meat diet. Moreover, discontinuation of dietary red meat reduced plasma TMAO to the initial values within 4 weeks.

Other studies have not found an association between the intake of meat and TMAO levels. A dietary intervention study was carried out to discover exposure markers in urine after consuming different test meals prepared with three protein sources (meat, fish or vegetarian). ${ }^{88}$ No clear difference in TMAO was observed between meals prepared with vegetarian or a meat source; conversely, TMAO was found as a fish consumption marker. In different cross-sectional study, Kühn et al. (2016) did not observe a clear association between meat consumption and fasting plasma levels of TMAO. ${ }^{89}$ By contrast, TMAO concentrations showed clear intra-individual variation over time, presumably driven by a greater within-person than between-person variation. Similarly, Rohrmann et al. (2016) did not observe any associations between the consumption of meat (red, processed and white), fish, eggs and the plasma concentrations of TMAO. ${ }^{90}$ However, participants with higher consumption of total milk and dairy products (but not cheese) showed higher plasma TMAO concentrations. ${ }^{90}$ Concerning this issue, recently, it has been suggested that fermented dairy products have different effects to those of nonfermented dairy products on postprandial TMAO levels. Thus, Burton et al. reported that fermented milk products were associated with lower postprandial TMAO responses than nonfermented dairy consumption; on the other hand, daily consumption of dairy products did not differentially affect fasting. TMAO. $^{91}$

As can be appreciated, there is high heterogeneity in the described associations between meat intake and TMAO. This can be potentially explained due to differences in the study duration, quantity of food dosing, variability in L-carnitine/ choline content different types of tested meats, and the small number of participants included in some of the studies. Thus, careful attention should be paid when interpreting variations in urinary or plasma TMAO levels in diverse populations. Furthermore, the link between measured TMAO and dietary 
TMA-rich foods could also be confounded by the large degree of inter-individual variability in gut microbiota composition. It seems reasonable to think that, considering the primary role of microorganisms in TMA generation, gut microbiota composition and community structure exert a strong influence in circulating TMAO levels. In line with this, some studies have suggested that gut microbiota composition influences the extent of TMAO production in dietary intervention studies. ${ }^{69,92}$ Cho et al. compared TMAO response for eggs, beef, and fruit control taken in a single day, and a greater increase in postprandial plasma TMAO was detected for both eggs and beef compared to the fruit control. ${ }^{69}$ They observed that highTMAO producers, i.e. those subjects having $\geq 20 \%$ increase in urinary TMAO concentrations following the consumption of egg or beef meals, had more Firmicutes than Bacteroidetes and a less diverse gut microbiome. More specifically, highTMAO producers were represented by Clostridiales at the Firmicutes phylum, within which Clostridiaceae, Lachnospiraceae, and Veillonellaceae were the most abundant. On the other hand, low-TMAO producers $(<20 \%$ increase in urinary TMAO concentrations) were represented by Bacteroidales within the Bacteroidetes phylum, of which Bacteroidaceae and Prevotellaceae were predominant. These findings agreed with those obtained by Falony et al. (2015) suggesting that TMA production function is widespread in Firmicutes, Proteobacteria, and Actinobacteria, whereas it appears to be absent in Bacteroidetes. ${ }^{40}$ In another randomized, controlled crossover design, changes in the gut microbiota composition were observed after 4 weeks of dietary intervention that varied in the protein source (lean-seafood or nonseafood). ${ }^{92}$ A higher fecal level of TMA was observed after consumption of the lean-seafood diet compared to the nonseafood diet. Moreover, a trend toward a higher relative abundance of Firmicutes with an accompanying decrease in the relative abundance of Bacteroidetes was observed after the non-seafood diet. On the contrary, the ratio of Firmicutes to Bacteroidetes was maintained during the lean-seafood intervention.

Resistant starch has shown potential to exert an impact on the microbial ecosystem of the gut and its composition, since the gut microbiota uses dietary fiber as an energy source. Under this assumption, Bergeron et al. studied the effect of dietary resistant starch (RS) in the gut microbiota-derived metabolite TMAO in plasma. In a controlled, randomized, crossover dietary intervention, they observed that fasting plasma levels of TMAO were significantly increased by high $v s$. low RS intake, although this effect was dependent on total dietary carbohydrate intake. ${ }^{93}$ Microbial composition of fecal samples showed that the proportions of certain taxa were correlated with plasma TMAO levels; however, results about microbiota composition were inconclusive.

\subsection{Association of TMAO levels with dietary patterns}

There is also a large interest in examining the effect of diet as a whole instead of intake of single TMA-enriched foods or nutrients. Some studies have attempted to associate the con- sumption of specific dietary patterns and health outcomes with plasma TMAO. Although there are still only few works, some of them have shown that dietary habits and patterns have the ability to influence circulating dietary trimethylamines as well as their microbial metabolites TMA and TMAO.

Western lifestyle, including diet, may predispose populations to chronic disease such as, CVD, colorectal cancer and type-2 diabetes (T2D). The Western diet frequently contains excessive saturated and trans fatty acids, animal protein content and simple sugars, and is low in fiber, vegetables and other plant-based foods. Moreover, some cross-sectional studies have shown evidence that high-protein and high-fat diets (related to Western lifestyle) are associated with gut microbial populations that are typified by a Bacteroides enterotype; whereas diets rich in carbohydrates and simple sugars are associated with a Prevotella enterotype. ${ }^{94}$ Western-style diets or high-fat diets have shown to increase plasma TMAO levels. In a short-term (5 days) interventional trial, high-fat diet (55\% fat) administered to healthy men did not increase fasting plasma TMAO concentrations, but led to increase TMAO in the postprandial period. ${ }^{95}$ Slightly higher concentrations of L-carnitine, choline and TMAO were also found in those persons who had more Western-style dietary patterns. ${ }^{96}$ O'Sullivan et al. (2011) pioneered the use of cluster analysis for the identification of dietary patterns. ${ }^{97}$ In their work, three dietary clusters were identified on the basis of the energy contribution of different food groups. Cluster 3 (related to Western-style diet) had higher intakes of red meat, meat products and a lower contribution from vegetables, while Cluster 1 presented the highest intakes of vegetables, wholegrain breads, and fish. The key metabolites in urine responsible for the separation of dietary Clusters 1 and 3 were TMAO, glycine, $\mathrm{O}$-acetylcarnitine, and phenylacetylglutamine.

Epidemiological studies have shown that the Mediterranean diet (MD) is associated with a lower risk of CVD and certain types of cancer. ${ }^{98} \mathrm{MD}$ is characterized by abundant plant-based foods (fruit, nuts, vegetables, legumes, cereals), olive oil as the principal source of fat, a high to moderate intakes of fish, a moderate or low consumption of eggs, poultry and dairy products (principally cheese and yogurt), a low consumption of processed meats, red meat and sweets, and also, wine consumed in low to moderate amounts, normally with meals. ${ }^{99,100}$ Beneficial effects of MD have been attributed to the types of food consumed, total dietary pattern, as well as other aspects such as, eating and lifestyle behaviors. ${ }^{98}$ Dietary interventions with MD have shown an inverse association between adherence to the MD and cardiovascular risk. The PREDIMED (PREvention with MEDiterranean DIet) was a pioneering multicenter, randomized, nutritional intervention trial, for the primary prevention of CVD with MD. The study was carried out in Spain from 2003 to 2011. In this study, participants who were at high cardiovascular risk (but with no CVD) received a dietary intervention with MD supplemented with extra-virgin olive oil (EVOO), a MD supplemented with nuts, or a control diet (with an advice on a low-fat content). Among persons at high cardiovascular risk ( $n$ 
$=7447)$, MD supplemented with EVOO $(n=2543)$ or nuts $(n=$ $2454)$ reduced the incidence of major cardiovascular events. ${ }^{101}$ In a sub-population of the PREDIMED trial $(n=98)$, the effect of MD pattern on urinary metabolome was assessed by comparing subjects at 1 and 3 years of follow-up, after an MD supplemented with either EVOO or nuts, to those on advice to follow a control low-fat diet. By means of a non-targeted metabolomics approach after dietary intervention it was observed that a low-fat diet was associated to an increased $24 \mathrm{~h}$ urine hippurate and TMAO levels, compared with a MD supplemented with EVOO. ${ }^{102}$ In a different work, 119 individuals from PREDIMED trial were studied, and according to their individual MD adherence based in PREDIMED 14-item questionnaire, ${ }^{103}$ they were assigned to low ( $\leq 7$ score) or high $(\leq 10$ score) adherence to MD. ${ }^{104}$ Thirty-four metabolites were identified in urine as discriminant between low-MD and high-MD adherence. Surprisingly, among them, both dimethylamine and TMAO were higher in the high-MD adherence group, and authors associated this result to a higher fish intake in the high-MD adherence diet.

Other studies different from PREDIMED, have also evaluated the association between levels of TMAO and the adherence to the MD. In a cross-sectional observational study Barrea et al. (2019) used the PREDIMED 14-item questionnaire to assess the adherence to the $\mathrm{MD},{ }^{105}$ and association between levels of TMAO and the MD in 144 healthy adults was found, with a clear gender difference in this association. ${ }^{106}$ De Filippis et al. (2016) observed a cohort of 153 healthy volunteers comprising 51 vegetarians, 51 vegans and 51 omnivores, of which $30 \%$ had a high adherence to MD. ${ }^{107}$ They found that the lower adherence to the MD group was related with a higher urinary TMAO levels. Associations were also found between TMAO levels and microbial genera that were more linked to the intake of animal proteins and fat. Ruminococcus genus from the Lachnospiraceae family was positively associated to TMAO. Interestingly, Griffin et al. (2019) showed that six months of dietary intervention with MD did not alter fasting TMAO concentration in a population of 115 healthy people at increased risk of colon cancer. ${ }^{108}$ It was suggested that broad dietary pattern intervention over six months may not be sufficient for reducing TMAO concentrations. Also, Pignanelli et al. (2018) did not find any significant difference in the plasma levels of TMAO and other gut-derived uremic toxins associated with a MD score or with intake of dietary precursors among omnivorous vascular patients. ${ }^{109}$

\section{Intervention strategies for targeting TMA/TMAO pathway}

In last years, the recognition of mechanistic links between TMAO and pathogenic molecular processes associated with atherosclerosis and thrombosis has placed this microbial metabolite on the map as an emerging therapeutic target for the prevention and treatment of CVD. Considering the multifactorial nature of TMAO generation, various strategies may be envisaged to interfere with the different biochemical and physiological steps that constitute the metaorganismal TMA/TMAO pathway in order to reduce circulating TMAO levels and prevent or reduce the risk of CVD (Fig. 2). In this context, limiting the dietary consumption of food enriched in dietary trimethylamines, such as some animal source foods, could be a straightforward point of intervention; however, this would entail adverse clinical consequences since, as discussed above, they contain nutrients that are required for optimal health. Another option for intervention is the inhibition of hepatic FMO3 activity. It has been pointed out that this might not be a suitable approach, as impaired FMO3 activity gives rise to the inherited disorder trimethylaminuria (TMAU), a condition called Fish odor syndrome. Affected individuals cannot produce TMAO and, consequently, excrete large amounts of malodorous TMA in their urine, sweat, and breath. ${ }^{110}$ On the contrary, hepatic FMO3 is increased in insulin-resistant mice and humans, ${ }^{111}$ and recent research by Chen et al. (2019) suggests that FMO3 inhibition could be a suitable target to control TMAO production. ${ }^{34}$ Their study provided interesting data showing that FMO3 inhibition by dietary 3,3'-diindolylmethane or indole-3-carbinol (phytochemicals in cruciferous vegetables such as cauliflower and Brussel sprouts) supplementation reduced hepatic TMAO in insulin-resistant mice leading to a concomitant decrease in activated PERK and FoxO1, and improved glucose tolerance. It was reasoned that partial inhibition of FMO3 activity could be a tradeoff for having the beneficial TMAO-lowering effects without causing odor.

Alternatively, the discovery of microbiota as a major factor determining the amount of TMA generated in the gut has raised the possibility for devising strategies to modulate gut microbiota composition and its metabolic function. A large body of literature has demonstrated that administration of antibiotics can markedly decrease circulating TMAO levels. However, the transient nature of the changes in circulating TMAO levels, the various undesirable side effects and the serious risk for antibiotic resistance associated with the use of antibiotics are some of the reasons for dismissing their use as a therapeutic strategy to modulate circulating TMAO levels. In line with the microbiota obliged role in TMA formation, remodeling gut microbiota composition or its metabolic function by repopulating gut with other microorganisms could be a feasible alternative to decrease circulating TMAO levels. In this regard, fecal microbiota transplantation (FMT) has been successfully used to prove the transmissibility of atherosclerosis susceptibility $^{112}$ and elevated platelet reactivity and thrombosis potential $^{113}$ in animal models. By contrast, in a double-blind randomized controlled pilot study in metabolic syndrome patients, the FMT from single lean vegan-donor did not altered TMAO levels or parameters related to vascular inflammation, in spite of changes in intestinal microbiota composition were detected. ${ }^{114}$ These contrasting outcomes reflect that FMT as therapy for treating CVD is still in its infancy and further research is needed to test its efficacy and safety for reducing CVD risk. ${ }^{115}$ 


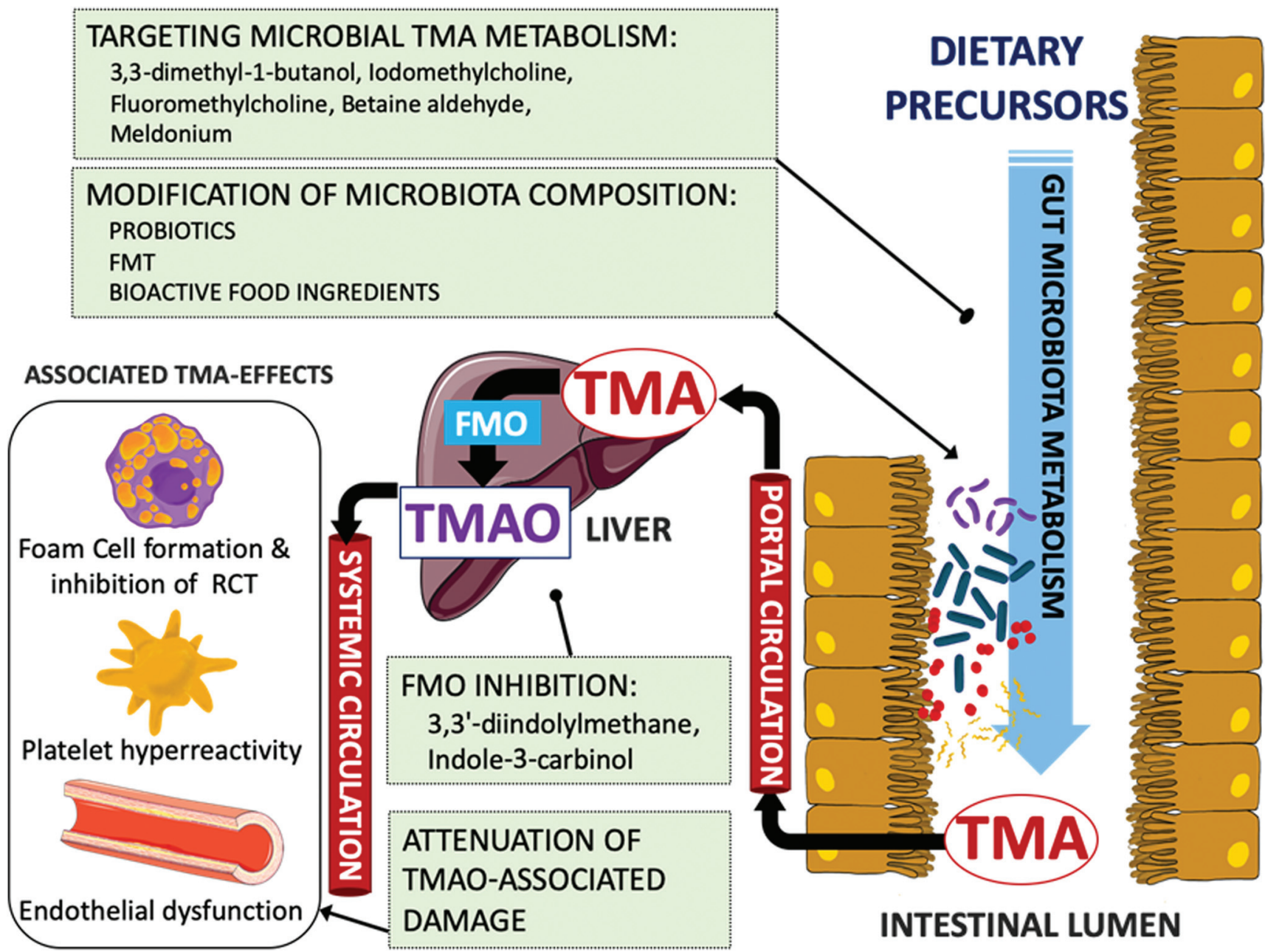

Fig. 2 Scheme of TMA/TMAO metaorganismal pathway and suggested intervention targets (green boxes).

Another strategy to alter microbiota composition is oral administration of probiotics. The idea of using living microorganisms that have a beneficial effect on health to repopulate the gut microbiota and reduce the extent of atherosclerosis is very attractive since it is thought to be non-invasive and to have little or no side effects. The various possible mechanisms by which probiotics could modulate the microbial metabolism and antagonize other strains to attenuate CVD have been recently reviewed by Din et al. (2019). ${ }^{116}$ Various oral probiotics have been evaluated for their ability to modulate the circulating TMAO levels in different cohorts including healthy subjects or patients with a CVD-related pathology. ${ }^{117-120}$ However, none of them seemed to significantly alter TMAO levels in the treated groups compared to the respective placebo groups. Other strategies involving the oral intervention with bacteria have been explored for the ability of specific strains to target TMA biosynthesis in the gut. For instance, Matsumoto et al. (2017) investigated the effects of Bifidobacterium animalis subsp. lactis LKM512 supplementation during 12 weeks in the fecal TMA content of healthy volunteers. ${ }^{121}$ Authors observed a reduction in fecal TMA concentration in the group sup- plemented with the probiotic that was coincident with a lower relative abundance of specific bacterial groups, including TMA-producing bacteria. Qiu et al. (2017) have explored another interesting strategy aimed at depleting TMA levels in the gut using TMA-degrading bacteria. In their report, oral administration of a TMA-metabolizing strain (Enterobacter aerogenes ZDY01) reduced the levels of TMA in cecum and TMAO in serum, and altered microbial community composition in mice. ${ }^{122}$ Despite these encouraging results, human intervention studies showing the efficacy of probiotics to reduce plasma TMAO are still lacking.

A specially promising strategy is represented by the oral administration of compounds that are able to specifically inhibit microbial TMA biosynthesis in the intestinal lumen. This targeted approach has been explored to discover structural analogs of choline to inhibit choline TMA lyase, a major player in TMA biosynthesis. The 3,3-dimethyl-1-butanol (DMB), naturally found in olive oil and grapeseed oil, was the first molecule to specifically inhibit choline TMA lyase in vitro and $e x$ vivo on fecal suspensions without exhibiting lethal effects on targeted bacteria. ${ }^{41}$ In addition, co-feeding experi- 
ments with $1 \%$ choline supplement and 1\% (v/v) DMB in the drinking water showed that exposure to DMB significantly reduced the circulating levels of TMAO in C57BL/6J mice, attenuating macrophage foam cell formation and atherosclerosis induced by high-choline diet. Further advances of the same group in this research line were directed to explore other possible benefits of targeting microbial TMA biosynthesis for reducing CVD risk, such as for instance the attenuation of thrombosis potential. ${ }^{123}$ In this case, various novel choline TMA lyase inhibitors, named halomethylcholines, were designed and developed to fulfill various criteria to act as suicide-substrates. The rationale behind this was that upon recognition and cleavage by the enzyme, the analog would be transformed in a highly reactive product that could lead to the irreversible inactivation of the enzyme. Using this approach, iodomethylcholine and fluoromethylcholine were found to exhibit the most potent inhibitory activity as determined in lysates from Proteus mirabilis with reported $\mathrm{EC}_{50}$ values of $1.3 \mathrm{nM}$ and 900 $\mathrm{pM}$, respectively, and greatly exceeding the observed $\mathrm{EC}_{50}$ for DMB under similar experimental conditions (i.e., $100 \mu \mathrm{M}$ ). It was also shown that choline TMA lyase inhibition in mice fed a high-choline diet reduced circulating TMAO levels and platelet aggregation. In a recent report, a structure-guided approach has been applied to discover choline and betaine analogues able to inhibit choline TMA lyase. ${ }^{124}$ In their interesting report, authors detailed the mechanism of interaction between the enzyme and the inhibitor betaine aldehyde, a molecule found in celery and other foodstuffs. The inhibition of TMA formation was demonstrated in vitro with a recombinant choline TMA lyase from Desulfovibrio alaskensis G20, in whole cell assays with various choline-metabolizing human gut isolates, and ex vivo with human fecal suspensions. Besides choline TMA lyase, other enzyme activities are known to take part in the microbial production of TMA in the gut, and thus, could also be susceptible for inhibition. In this regard, the anti-ischemic drug meldonium has been suggested to inhibit endogenous L-carnitine biosynthesis and enhance renal excretion of TMAO. ${ }^{67}$ Further in vitro studies have shown that this analog of L-carnitine and $\gamma$-butyrobetaine is also able to suppress L-carnitine transformation into TMA by the intestinal TMA-producer Klebsiella pneumoniae without affecting bacterial uptake of L-carnitine and growth, which may be suggestive of Rieske-type carnitine monooxygenase inhibition. ${ }^{125}$ These findings pave the way for the discovery of new candidate molecules that may serve in interventions to interfere with TMA biosynthesis in the gut lumen, and subsequently, reduce circulating TMAO levels and its linked deleterious effects on health.

As discussed previously, some investigations about the influence of dietary TMA precursors (and their rich food sources) on TMAO levels have resulted in mixed evidence from observational and intervention studies (summarized in Table 1). However, the generalized idea is that high intake of food enriched in TMA precursors has the potential to increase circulating TMAO levels. Also, the frequent observation that certain dietary patterns that include abundant intake of plant- based foods may reduce circulating TMAO levels suggests the possibility that plant-derived dietary constituents, other than TMA precursors, may also interfere with the TMA/TMAO metaorganismal pathway and affect the TMAO levels. Sustaining the hypothesis that certain dietary compounds may modulate the gut microbial composition and metabolism, the search for novel food ingredients that target the gut microbiota components and/or its TMA metabolic capacity offers great opportunities to devise novel functional foods to control TMA production and circulating TMAO levels.

\section{Dietary intervention with bioactive food ingredients and natural extracts}

Given the demonstrated relationship between diet, microbial TMA metabolism, and atherosclerosis promotion, the possibility that some dietary constituents with proved cardioprotective activities may also modulate circulating TMAO levels cannot be totally dismissed as these aspects have been poorly investigated. In this area, in vitro studies evaluating the potential of different dietary phytochemicals to interfere with microbial TMA generation have provided interesting data that bring to light the difficulties to investigate the complex link between diet and gut microbial metabolism. ${ }^{126}$ As it will be discussed next (and summarized in Table 2), a number of studies have been directed to investigate the effects of food constituents or plant-based extracts on circulating TMAO levels and/or the associated damage without examining possible implications in gut microbiota composition and metabolism. Alternatively, other studies on food ingredients and phytochemicals with TMAO-reducing activity have also included interesting data on different taxa of gut microbiota associated with the observed TMAO changes. In most of the cases, such associations do not establish a causative role of those taxa in TMAO changes, although such findings might offer great opportunities for hypothesis generation. In addition, most studies are currently carried out in animal models, and therefore, more translational research will be required to corroborate the efficacy of candidate dietary constituents in humans, and their observed beneficial impact.

\subsection{Evidence from animal studies}

Connected with the idea that intervention with specific dietary constituents could be a feasible option to interfere with TMAO, and possibly contribute to prevent atherosclerosis, various published studies investigating the potential effects of different food constituents, including dietary fibers, oils, and phytochemicals individually or in extracts, on circulating TMAO levels and/or its effects in animal models, are next discussed.

Food ingredients and natural extracts that attenuate TMAOrelated damage. Several studies have reported the ability of various food supplements and natural extracts to ameliorate or suppress the plasma TMAO-induced deleterious effects in animal models. To facilitate functional interpretation, various 


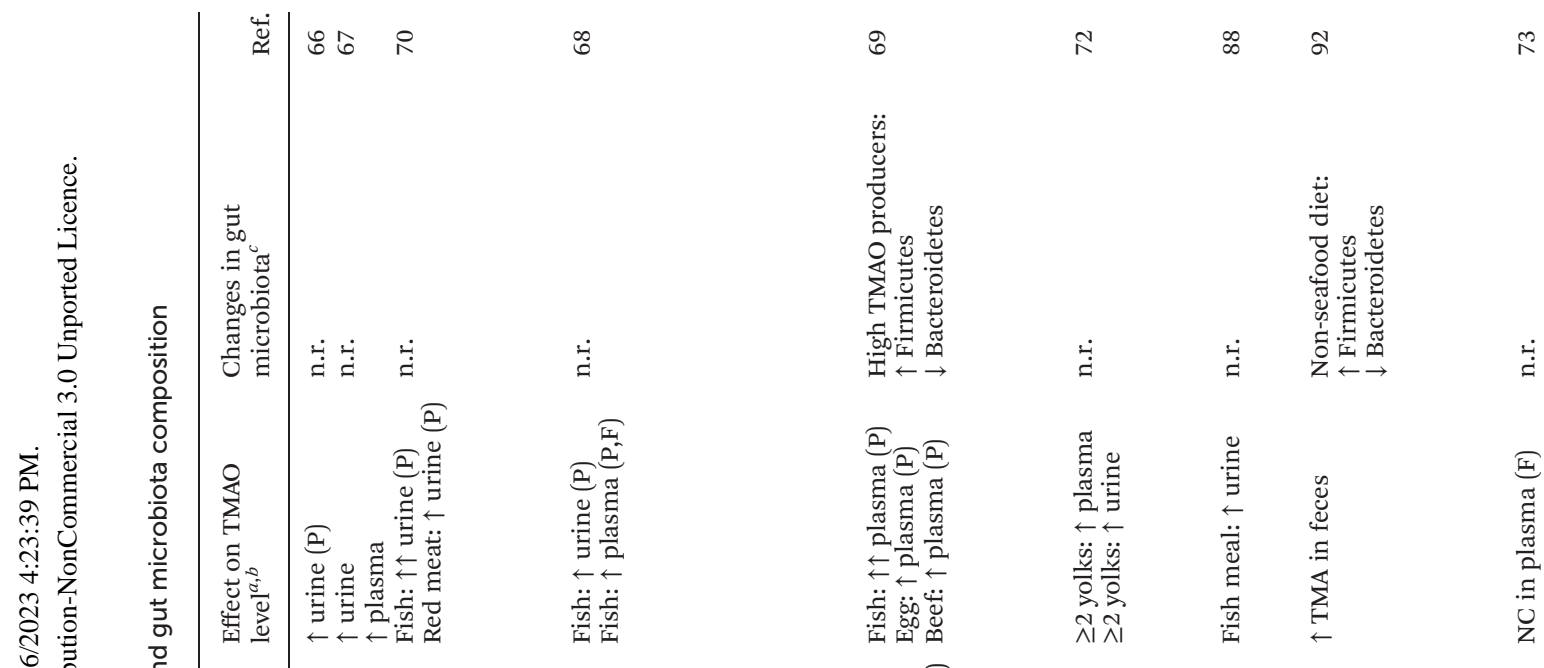

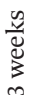

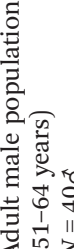

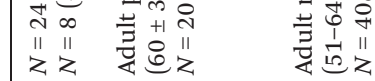

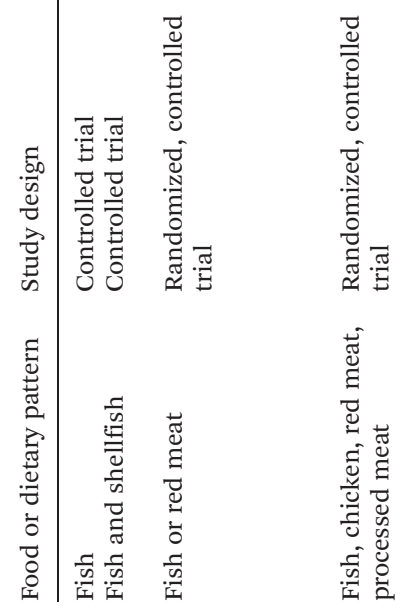

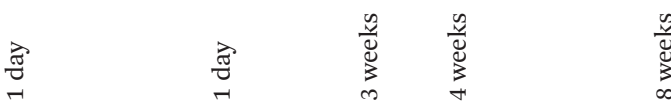
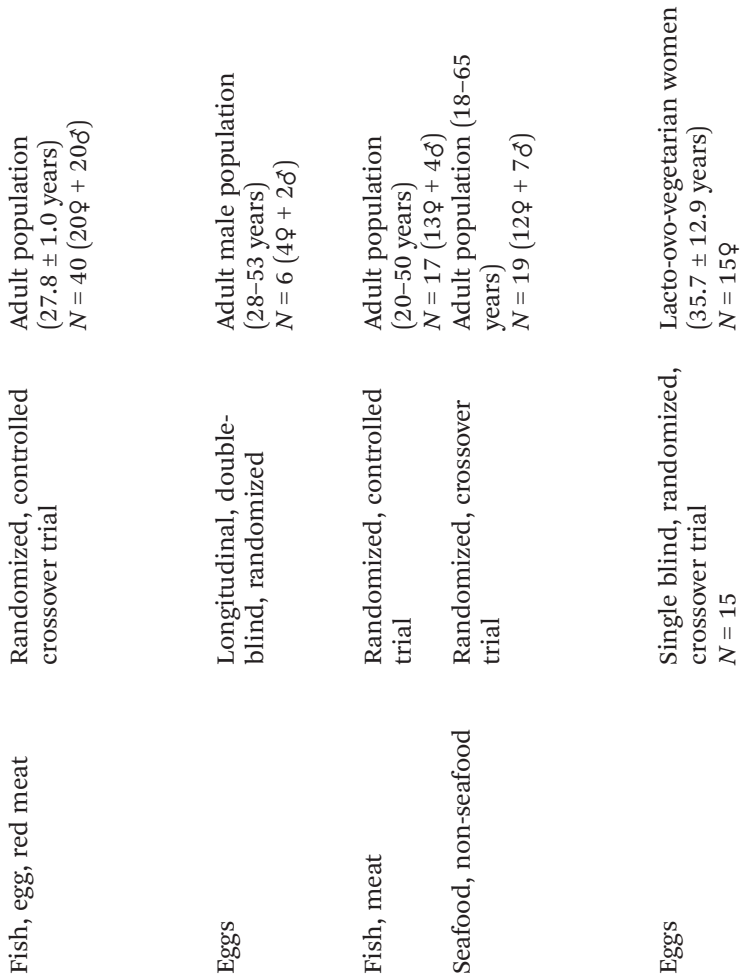

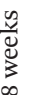

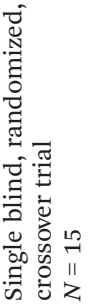

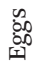




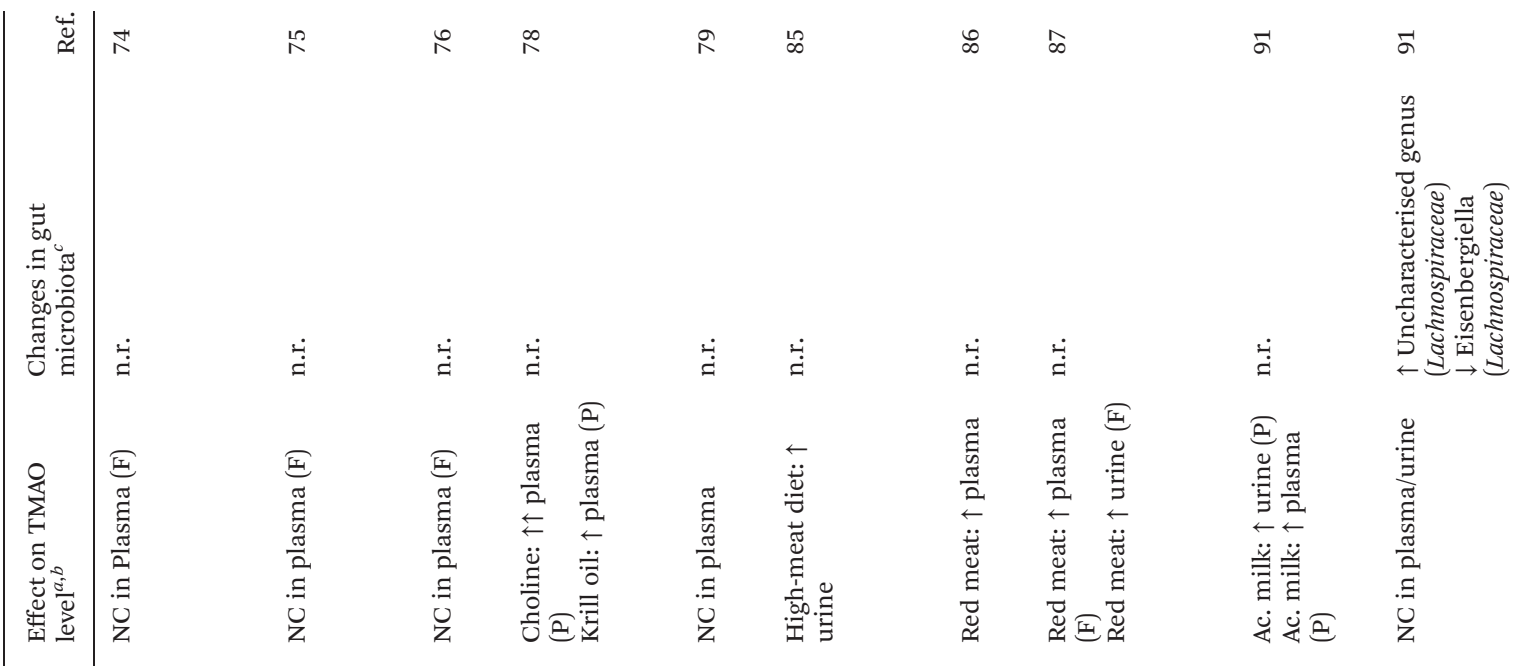

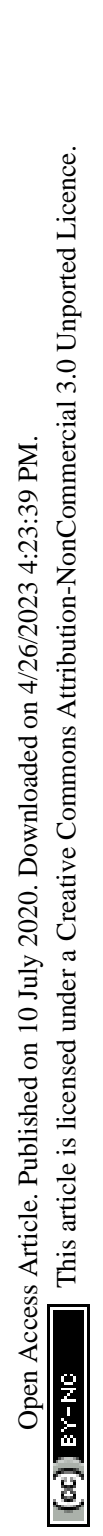

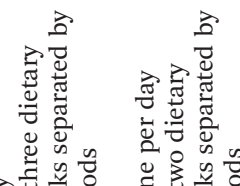

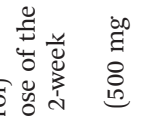

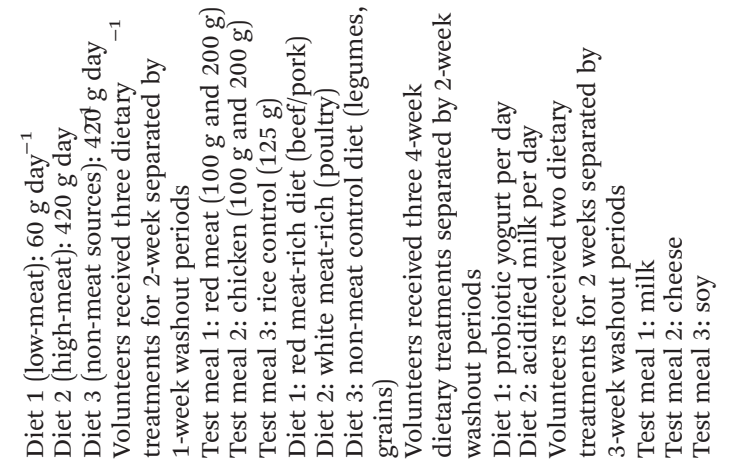

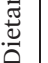

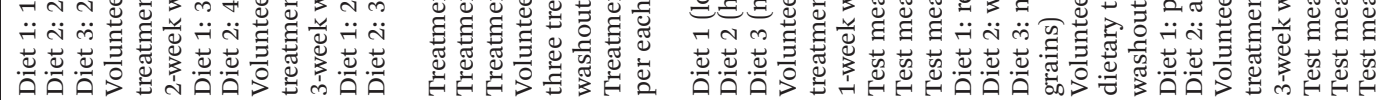




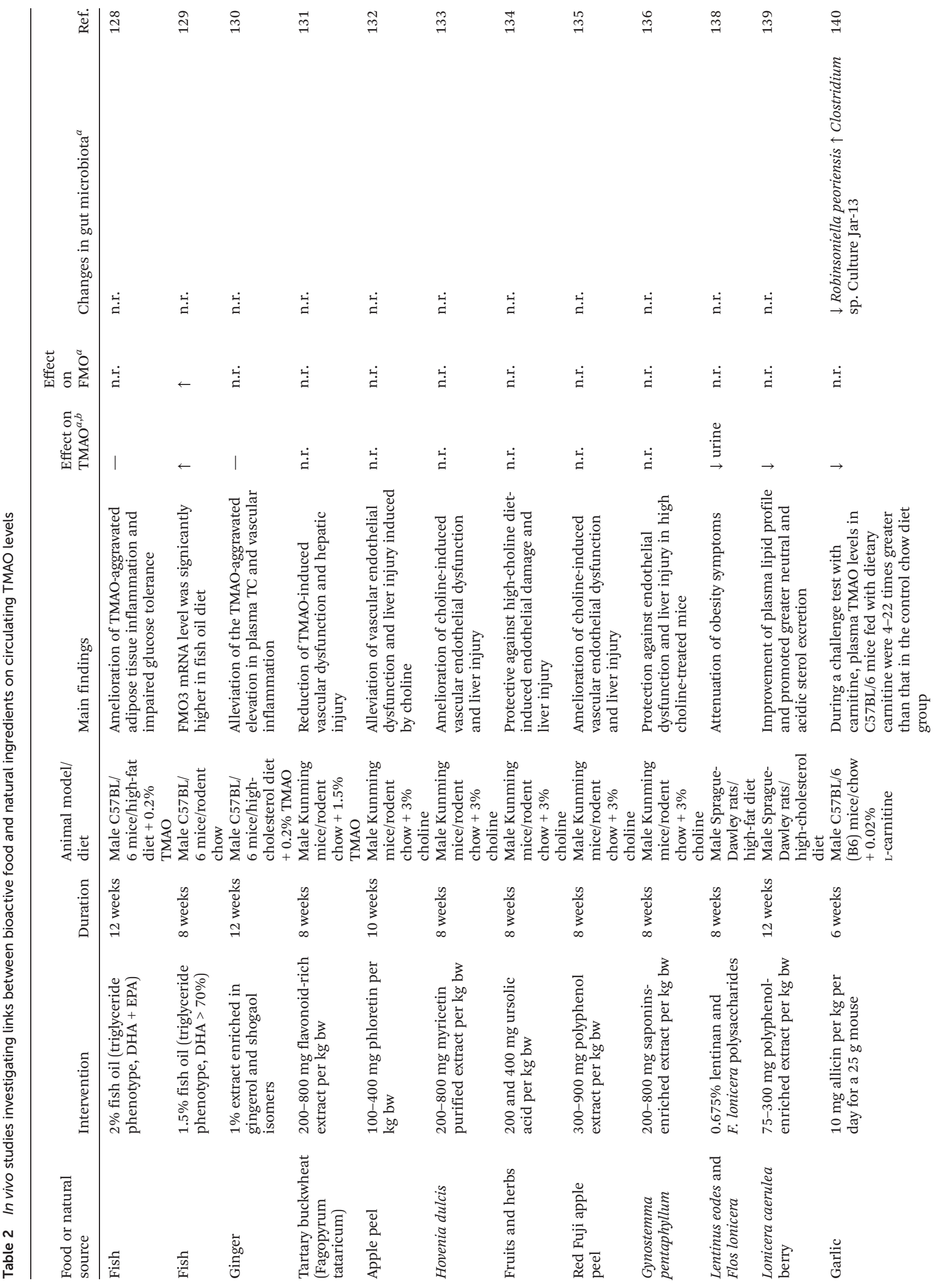




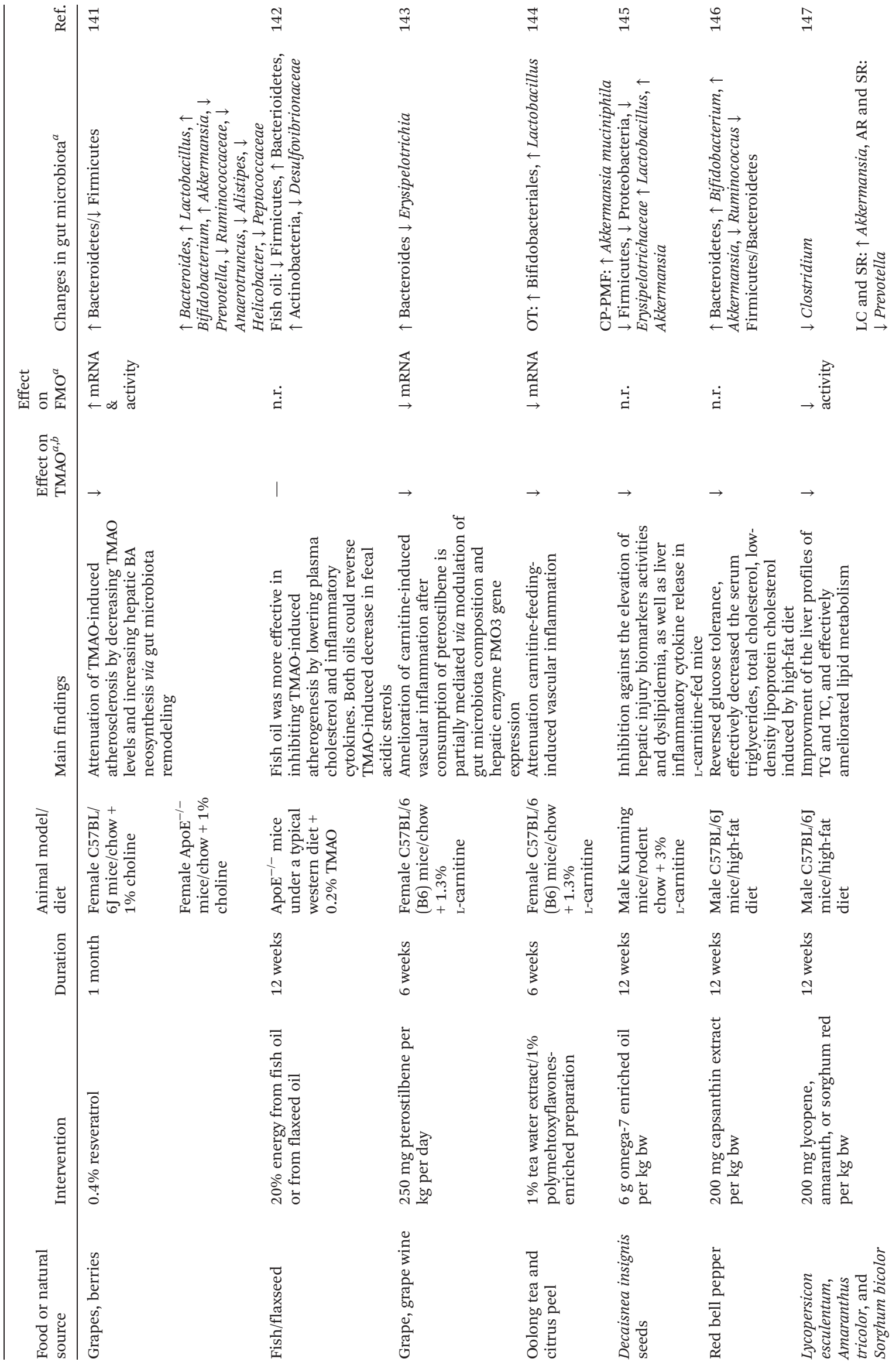


models of TMAO-induced damage have been utilized. Elevating plasma TMAO by direct oral intake of TMAO or supplements enriched in choline or $\mathrm{L}$-carnitine are the most common examples used to induce the development of different lesions. For instance, Gao et al. (2014) reported that $0.2 \%$ TMAO supplementation in high-fat diet-fed C57BL/ 6 mice for three weeks exacerbates impaired glucose tolerance, inflammation in adipose tissue and obstructs the hepatic insulin signaling. ${ }^{127}$ These observations were further confirmed by the same authors in another report, and suggested that the same dietary regimen but supplemented with $2 \%$ fish oil (DHA + EPA, 55\%) for 12 weeks can mitigate TMAO-aggravated glucose intolerance and also decrease adipose tissue inflammation. ${ }^{128}$ Interestingly, further research by the same authors suggested that 8-weeks supplementation with fish oil can transiently increase plasma TMAO levels via increased liver FMO3 activity after administration of TMAO precursors in mice. ${ }^{129}$ In a similar approach, He et al. (2018) reported that the exacerbated vascular inflammation and hypercholesteromic effects of adding 0.2\% TMAO in high-cholesterol diet-fed $\mathrm{C} 57 \mathrm{BL} / 6 \mathrm{~J}$ mice were ameliorated with the addition of $1 \%$ ginger extract enriched in gingerol and shogaol isomers. ${ }^{130}$ The cholesterol-lowering effect observed with dietary ginger extract was hypothesized to be the result of an increase in the synthesis and excretion of fecal bile acids. Although the report did not include gut microbiota data, it was speculated that the extract may reduce the number of coprostanol-producing bacterial species that could explain the observed changes of specific fecal neutral sterols. In a separate report, a 1.5\% TMAO was administered in drinking water to Kunming mice, allowing them free access to water and rodent chow for 8 weeks. ${ }^{131}$ Besides the control and TMAO-treated mice groups, three more mice groups under TMAO drinking water intake regime were administered intragastrically with different doses of a purified flavonoid-rich tartary buckwheat extract (enriched in rutin and quercetin). TMAO intake was reported to cause severe liver injury and vascular dysfunction that the flavonoid extract could prevent. In this study, the plasmatic TMAO were not reported, and authors suggested that the protective effect exerted by the tartary buckwheat extract may be mediated by inhibition of ROS generation and elevation of vascular nitric oxide production.

Although it is well known that lack of dietary choline can lead to non-alcoholic fatty-liver disease and muscle damage, a growing number of reports have demonstrated that ingestion of some choline-enriched diets can effectively induce TMAOassociated damage in mice, such as endothelial dysfunction and liver injury. In a series of works, Yang et al. have investigated the ability of various phytochemical-enriched natural extracts and food ingredients to reverse the harmful effects of chronic high-choline diet. More precisely, healthy male Kunming mice fed with regular chow supplemented with $3 \%$ dietary choline in water for 8 weeks served as experimental model for testing the protective potential of flavonoids, such as phloretin ${ }^{132}$ and myricetin purified extract from Hovenia dulcis Thunb.; ${ }^{133}$ other phenolic compounds such as the triter- pene ursolic acid ${ }^{134}$ and those found in red Fuji apple peel extract; ${ }^{135}$ and the saponin-enriched extract from Gynostemma pentaphyllum $^{136}$ against vascular dysfunction and hepatic damage. These co-feeding studies with the mentioned bioactive compounds and choline suggest that such phytochemicals have the potential to ameliorate the plasma lipid profile, hepatic function and biomarkers for endothelial function in mice. However, in spite of these observed beneficial effects, their underlying mechanism remain unclear and a link between them and TMAO damage cannot be directly established since TMAO levels in plasma and tissues were not reported. With regards to bioactive saponins, an in vitro study with HUVECs has recently demonstrated that an asparagus saponin-enriched extract reverses the inhibitory effect of TMAO on cell proliferation. ${ }^{137}$ Such effect appears to be mediated by Clock and Baml1 molecules, that play relevant roles in circadian cycle and are under control of NEAT1 and MAPK pathways. In contrast to other reports, TMAO exposure seemed to have a negative effect on MAPK signaling; however, such inhibitory activity was observed at TMAO concentrations that are well above the physiological circulating levels reported in humans and mice.

Accumulated evidence suggests that high-fat diet induces obesity concomitant with the rise of circulating TMAO levels in animal models. In a study with high-fat diet fed SpragueDawley rats, this model was established to evaluate the antiobesity effects of supplementing a mixture of lentinan $\beta$-glucan and Flos Lonicera polysaccharide after a 8-weeks period. ${ }^{138}$ In this case, untargeted NMR metabolomic analysis combined with multivariate analysis indicated that polysaccharide mixture intervention reduced the TMAO levels in urine compared to the levels found during the endogenous metabolic response to high-fat diet, suggesting that polysaccharides may also affect the structure of intestinal flora; however, further research would be necessary to verify such hypothesis. In male Sprague-Dawley rats, high-cholesterol diet also induced high levels of plasma TMAO in addition to hypercholesterolemia. ${ }^{139}$ Supplementing the diet with a polyphenolenriched extract from Lonicera caerulea berry attenuated such effects and increased fecal excretion of neutral and acidic sterols. Authors discussed that a plausible underlying mechanisms to explain the cholesterol-lowering effect of the extract involved inhibition of cholesterol absorption, mediated by downregulation of NPC1L1, ACAT2 and MTP, and upregulation of ABCG5/G8 transporters. However, the mechanism by which the extract induced plasma TMAO levels are unclear since gut microbiota data and hepatic FMO3 activity were not reported.

Bioactive ingredients that alter TMAO levels and affect gut microbiota. Collectively, the aforementioned studies provide encouraging results about the ability of food ingredients and natural extracts for alleviating TMAO-related harmful effects. However, our knowledge about the link between the observed effects by those individual compounds or mixtures on endogenous TMAO and the gut microbiota is very limited. In the last decade the investigation of the effects of dietary constituents, other than TMA precursors, on microbiota compo- 
sition, structure and metabolism has attracted much more attention; however, in the last three years is when a significant number of reports, directed to study potential microbiotamediated mechanisms underlying the observed effects of bioactive dietary constituents (individually or in extracts) on TMAO levels have been published. One of the first reports aimed at investigating these aspects is the one related with dietary allicin and its potential to reduce the transformation of ingested L-carnitine into TMAO through modulation of gut microbial ecosystem in mice. ${ }^{140}$ Although in their study authors used synthetic allicin as supplement, this compound is naturally present in crushed garlic and it has a recognized broad spectrum antimicrobial activity. To test their hypothesis, authors performed experiments in male C57BL/6 mice with an amount of allicin, given via gastric gavage, equivalent to the amount that an adult human would acquire from 5 cloves of freshly blended garlic per day. Allicin supplementation was tested with two chow diets, the normal control and $0.02 \%$ L-carnitine in the drinking water, as calculated to be equivalent to the intake of an adult human following an omnivorous diet. After feeding mice for 6 weeks, a L-carnitine challenge test indicated that plasma TMAO increased up to 22 times greater in mice fed with dietary L-carnitine than that in the control chow diet, and more interestingly, allicin gavage in the L-carnitine supplemented diet fed mice counteracted the elevation of TMAO levels in plasma during the challenge. A comparison of microbiota composition in cecum in the different mice groups revealed differences in the relative abundance of some bacterial species, and the analysis of data suggested positive correlation of several species, including Robinsoniella peoriensis, with elevation of TMAO in plasma. Although, authors hypothesized that allicin may play as a Rieske protein inhibitor to reduce L-carnitine conversion to TMA by gut microbiota, various questions may remain opened, including a possible inhibitory effect of allicin of FMO3 activity.

The anti-atherosclerotic effects of resveratrol and their underlying mechanisms associated with microbiota composition have been investigated by Chen et al. (2016). ${ }^{141}$ In their study, authors found that addition of $1 \%$ choline in chow diet during 30 days significantly increased the plasmatic levels of TMA and TMAO in female C57BL/6J mice, whereas the same diet supplemented with $0.4 \%$ resveratrol reversed the elevation of both metabolites. However, this effect was transient since a washout with a chow diet for 1 month after the treatment failed to reduce TMA and TMAO in the same mice. Interestingly, resveratrol supplementation appeared to increase hepatic FMO3 activity, indicating that the observed resveratrolinduced decrease in TMAO levels was not mediated by FMO3 inactivity in the liver, and therefore, suggesting a possible involvement of gut microbiota as mediator of the resveratrol effects. Reinforcing that hypothesis, ex vivo experiments with cecal content from resveratrol-treated mice showed a lower capacity to produce TMA from choline than in the control nonsupplemented mice. Analysis of gut microbiota composition revealed that the choline-enriched diet supplemented with this phytochemical induced significant changes at the phylum level showing an evident decrease of the Firmicutes to Bacteroidetes ratio, and at genus level, resveratrol increased the relative abundance of genera, Bacterioides, Akkermansia, Lactobacillus and Bifidobacterium, the two latter being related to bile salt hydrolase activity, bile acids deconjugation and fecal excretion. Connected to this, bile acid content in ileum was reduced, an effect that was coincident with the down-regulation of the gut-liver FXR-FGF15 axis and the activation of bile acids synthesis.

More recently, a comparison of the effects of fish oil and flaxseed oil on gut microbiota of $\mathrm{ApoE}^{-/-}$mice under a typical western diet supplemented with TMAO for 12 weeks has recently been reported. ${ }^{142}$ The experimental design included various diets, including a Western diet, a WD with added $0.2 \%$ TMAO, and two experimental WDs with added TMAO and with half of the lard being replaced by either flaxseed oil or fish oil for 12 weeks. In agreement with previous studies, results showed that addition of $0.2 \%$ TMAO to WD increased by 2 -fold circulating plasma TMAO, and significantly aggravated atherosclerotic lesions. Neither flaxseed oil nor fish oil significantly altered the plasma TMAO concentration; however, both flaxseed oil and fish oil could reverse the reductions in fecal acidic sterol excretion and CYP7A1 expression induced by the TMAO addition to WD. On the other side, only fish oil feeding had a significant effect facilitating the fecal excretion of neutral sterol and could significantly lower the plasma concentration of proinflammatory cytokines. Amplicon sequencing analysis of 16S rDNA gene suggested that TMAO addition into Western diet did not lead to an obvious shift in overall microbial profile, whereas both flaxseed oil and fish oil had great impacts on the microbial profile composition. For instance, feeding fish oil led to a sharp decrease in the Firmicutes to Bacteroidetes ratio, and featured an increased population of Actinobacteria compared with feeding western diet with TMAO. Fish oil was more effective than flaxseed oil in promoting the growth of short-chain fatty acid-producing bacteria and lowering microbial generation of lipopolysaccharides. Thus, it could be concluded that, in general, fish oil exerted more potent cardioprotective effects than flaxseed oil in counteracting TMAO-exacerbated pro-atherosclerotic effects.

More recently, Koh and colleagues reported the effect of pterostilbene, a dimethoxylated analog of resveratrol found in a wide variety of dietary sources, on alleviating TMAO generation and its harmful consequences in vascular function. ${ }^{143}$ In this case, addition of $1.3 \% \mathrm{~L}$-carnitine water to chow diet increased plasma TMAO up to eight-fold in C57BL/6 mice. Supplementation of the high-carnitine diet with $0.05 \%$ pterostilbene partially suppressed TMAO elevation compared to the observed in the high-carnitine control group. Pterostilbene also significantly down-regulated FMO3 gene expression compared with the FMO3 gene expression level observed in mice fed high-carnitine diet, a finding that may also explain the reduced plasma TMAO observed in mice fed pterostilbene supplemented high-carnitine diet. In addition, 16S rRNA sequencing analysis revealed that supplementing diet with this poly- 
phenol increased the relative amount of Bacteroides and decreased Turicibacter, a genus belonging to Erypsipelotricia, recently associated with elevated TMAO. To note, authors discussed that, in agreement with the recent report by Wu et al. (2019), ${ }^{137}$ measurements of CntA gene abundance in fecal samples did not provide a good correlation with TMAO levels, an observation that reflects the multifactorial nature of TMAO generation.

In another work, same researchers utilized the same experimental protocol to study the activities of Oolong tea water extract and citrus peel polymethoxyflavones-enriched preparation against TMAO formation and vascular inflammation induced by high-carnitine feeding. ${ }^{144}$ In this case, the experimental high-carnitine diet was supplemented with either $1 \%$ Oolong tea extract or $1 \%$ polymethoxyflavone preparation and utilized in mice feeding for 6 weeks. Similar results with regard to plasma metabolites and FMO3 gene expression as previously discussed with pterostilbene were obtained. More precisely, plasma TMAO levels in Oolong tea extract- and polymethoxyflavone-treated mice were significantly lower than those in the high-carnitine control diet group, but the plasma L-carnitine levels were more elevated. In addition, both supplements suppressed the FMO3 gene up-regulation observed in high-carnitine diet fed mice. Interestingly, cecum microbiota analysis suggested that carnitine group increased the relative abundance of Proteobacteria and Tenericutes and reduced the relative abundance of Deferribacteres, and at genus level, both supplements increased the relative abundance of Bacteroides compared to the high-carnitine group. In addition to this common effect to both supplements, in polymethoxyflavones-treated group the relative abundance of Akkermansia increased, whereas those of Lactobacillus and Bifidobacteriales increased only in the Oolong tea-treated mice, changes that were similar to the observed effects exerted by resveratrol, as reported by Chen et al. $2016 .{ }^{141}$

Decaisnea insignis seed oil mainly consists of monounsaturated fatty acids, and it has been reported to have a similar fatty acid profile as olive oil. Zhang et al. (2019) have recently investigated the effectivity of this oil in alleviating liver dysfunction induced by high-carnitine diet in mice. ${ }^{145} \mathrm{D}$. insignis seeds were extracted using supercritical fluid extraction, and the obtained oil was used to supplement normal diet with $3 \%$ L-carnitine in drinking water in Kunming male mice for 12 weeks. Chronic administration of such high-carnitine diet elevated plasma and urinary TMAO and TMA levels and induced abnormal liver lipid metabolism compared with normal diet. On the other side, diet with $D$. insignis seed oil decreased TMAO levels and improved hepatic serum lipid and liver injury biomarkers in high-carnitine diet fed mice. Moreover, $D$. insignis seed oil consumption affected the total SCFA content in high-carnitine diet fed mice. Another interesting counteracting effect of the seed oil extract treatment were observed in microbiota composition. For instance, it restored the unbalance exerted by high-carnitine diet at the phylum level, specially decreasing the Firmicutes to Bacteroidetes ratio. Also the relative abundance of Proteobacteria in high- carnitine diet was significantly decreased by seed oil administration. Interestingly, at the genus level, seed oil treatment increased proportions of Akkermansia, Bacteroides, and Lactobacillus in high-carnitine diet-fed mice, whereas Erypsipelotrichaceae abundance was reduced. In addition, the latter genus showed significant positive correlation with increased serum TMAO levels.

As mentioned above, compelling evidence suggests that TMAO regulates cholesterol and sterol metabolism in various tissues and processes, thus having a putative role in obesity. Connected with this, $\mathrm{Wu}$ and colleagues investigated whether the anti-obesity effect of various food ingredients and extracts are associated with gut microbiota and TMAO using high-fat diet-induced obese $\mathrm{C} 57 \mathrm{BL} / 6 \mathrm{~J}$ mice. ${ }^{146,147}$ In one report, authors extracted red bell pepper using supercritical fluid extraction to obtain a product containing $92.5 \%$ capsanthin, a carotenoid pigment with reported anti-inflammatory activity. ${ }^{146}$ Capsanthin-enriched extract was added to high-fat diet and administered for 12 weeks. Capsanthin extract alleviated the high-fat diet-induced high blood glucose level and dyslipidemia, decreased by $31 \%$ serum TMAO level and reduced body weight by $27.5 \%$ compared with untreated highfat diet-fed mice. Furthermore, capsanthin modestly reduced the relative abundance of Firmicutes and increased that of the Bacteroidetes in the high-fat diet group. More importantly, capsanthin-enriched extract consumption increased some genera including Allobaculum, Akkermansia, Bifidobacterium, Blautia, Coprobacillus, whereas the relative abundance of some other declined, including Lactobacillus, Prevotella, Helicobacter, Bacteroides, Ruminococcus, Parabacteroides, Odoribacter and Bilophila, suggesting that capsanthin-enriched extract modulated the gut microbial environment of high-fat diet-fed mice. Authors hypothesized that observed decrease in serum TMAO level with capsanthin treatment could be due to changes in the intestinal microbiota environment caused by the weight loss in mice; however, as in other studies the influence of the extract on the liver FMO3 activity was not reported. In further research by the same group, the same experimental setting was utilized to study anti-obesity activity and association with gut microbiota structural changes and TMAO generation of other three food pigments. Thus, lycopene, amaranth, and sorghum red pigments were obtained in the laboratory from Lycopersicon esculentum (M.), Amaranthus tricolor, and Sorghum bicolor, respectively. ${ }^{147}$ The three pigments provided similar results as with capsanthin extract in terms of serum TMAO, glucose level and dyslipidemia, being shorgum red the least potent in lowering TMAO levels. After the administration of high-fat diet supplemented with lycopene, amaranth, or sorghum red, the intestinal microbial environment improved in terms of increased bacterial diversity. Lycopene and sorghum red favored the proliferation of Akkermansia, whereas amaranth and sorghum red limited the proliferation of Prevotella. Thus, similar conclusions and hypothesis with regard to TMAO metabolism were drawn; however, in this study, data indicated that supplementation with these phytochemicals significantly reduced liver FMO3 activity, suggesting 
that lower hepatic capacity to metabolize microbial TMA into TMAO may be possible.

Dietary fibers are known to provide direct physical benefits, including increased fecal bulking and laxation. A growing body of evidence suggests that certain dietary fibers have the ability to modify microbiota structure and function, and decrease CVD risk, which made them suitable supplements for functional food development. Among dietary fibers, watersoluble dietary fiber has been recognized to exert beneficial effects on health by different mechanisms. The effects of supplementation of soluble fibers on TMA/TMAO metabolism and the related gut microbiota have recently gained great attention. For instance, Cheng et al. (2017) observed that galacto-oligosaccharides plus inulin and ordinary dietary fiber, supplemented individually or mixed for three weeks, had different effects on the gut microecology in male BALB/c mice. ${ }^{148}$ Results revealed that functional oligosaccharides (galacto-oligosaccahrides and inulin) and ordinary dietary fiber supplementations gradually elevated TMAO levels over the experimental period, while the combination of both types of fibers favored reduction of TMAO levels in serum. That observation was coincident with the higher flora diversity found in supplementing diet with both fibers compared to individual supplementation and non-supplemented diets.

Li et al. (2017) investigated the effects of soluble dietary fibers on TMA and TMAO metabolism in C57BL/6 mice fed $20 \%$ red meat supplementation according to the Western-style diet. $^{149}$ In this report, chemically diverse arabinoxylanenriched fiber fractions obtained from natural, fermented (inoculating Rhizopus oryzae), and steam-exploded wheat bran, were added to Western-style diet at two different concentrations $(7.5 \%$ and $15 \%)$. The effects of the different supplements were investigated on parameters affecting host metabolism and gut microbiota structure after dietary intervention for 4 weeks. Results demonstrated that soluble fiber supplemented-diets could reduce TMA and TMAO metabolism by 40.6 and $62.6 \%$, respectively. Dietary fiber feeding, specially fermented wheat bran, changed gut microbial ecology and promoted the growth of certain beneficial bacterial species. Soluble fiber-diet improved the serum lipid profile, decreased energy intake, weight gain, and intestinal $\mathrm{pH}$ values. The characterization of microbial communities revealed some interesting associations, such as for example the occurrence of phyla that are negatively associated with TMA and TMAO production. Also, in one of the diets supplemented with fermented dietary fiber, the phyla positively associated with cholesterol metabolism was found to predominantly belong to Turicibacter and Bifidobacterium, which coincided with lower TMA and TMAO plasma concentrations. Other interesting findings were that diets supplemented with soluble fibers also increased the production of SCFAs with activation of the intestinal epithelial adenosine monophosphate-activated protein kinase (AMPK). Further research of this topic suggested that compared with red meat consumption, fiber consumption favored a shift in the mice gut microbiome towards increased production of butanoate, which was concomitant to up-regu- lation of genes that depend on AMPK signaling in the host intestinal epithelium. ${ }^{150}$

Fructus Ligustri Lucidi, coming from the dried ripe fruit of Ligustrum lucidum possesses a recognized anti-aging property to treat osteoporosis for a long time in Asia. A Fructus Ligustri Lucidi aqueous extract (enriched in flavonoids, terpenoids, phospholipids, and a long list of other classes of compounds) could have anti-osteoporotic effects and improve the cognitive function as observed in studies with chemically-aged male ICR mice with D-gal and $\mathrm{NaNO}_{2}$ for three months. ${ }^{151}$ Among other deleterious effects, aging mice presented significantly higher serum TMAO levels compared to those found in the normal controls. Interestingly, oral administration of the extract reduced serum TMAO to levels close to control diet, but also decreased hepatic FMO3 activity. In order to better understand the potential role of gut microbiota in the extract's efficacy against TMAO elevation and osteoporosis, authors compared normal control, aged control, and extract-induced gut microbiota changes in aged mice. Results revealed that gut microbiota in aged mice was characterized by abnormal changes in the Firmicutes to Bacteroidetes ratio, that were restored by the treatment with the fruit extract. Also, species of several bacterial taxa, including Sutterella, Unclassified_Clostridiales, Coprococcus, Clostridium, Aldercreutzia, Oscillospira, Desulfovibrio, Bifidobacterium, and Lactobacillus were associated with hepatic FMO3 activity and/or serum TMAO concentrations.

Yang et al. (2019) found that oral administration of Luhong granules, a complex mixture of various herb leaves, seeds, roots, flowers, and animal parts, for 6 weeks in acute myocardial infarction-induced Wistar rats could partially reverse ventricular remodeling and improve intestinal barrier function. ${ }^{152}$ To note, the serum TMAO and lipopolysaccharides levels in the untreated acute myocardial infarction-induced rats was increased, but the levels of both bacteria-derived products were decreased in the Luhong granules-treated rats. Amplicon sequencing of fecal material of untreated acute myocardial infarction-induced rats showed changes in Desulfovibrionaceae, Escherichia, Akkermansia, Bacteroidales, Alistipes, and Phascolarctobacterium, in which there are potential TMAO producers. Furthermore, Luhong granules-treated rat presented reduced relative abundance of Bacteroidales, Alistipes, and Phascolarctobacterium, three genera that can promote TMAO generation.

The flavonoid baicalin have shown to improve glycolipid metabolism and the production of SCFAs by remodeling the gut microbiota. Recently, the neuroprotective mechanism of orally administered baicalin has recently been explored in the mouse model of repeated cerebral ischemia-reperfusion injury, including the effects of baicalin on the gut microbiota. ${ }^{153}$ The reported results suggested that plasma TMAO levels were elevated in mice subjected to repeated cerebral ischemia-reperfusion, and baicalin supplementation suppressed cognitive and memory decline induced by repeated cerebral ischemia-reperfusion, restored functional connectivity and suppressed neuroinflammation. Interestingly, the results indicated that elev- 
ated plasma TMAO levels correlated with behavioral and electrophysiological deficits in the model group mice compared to non-ischemic control mice. The gut microbial composition in ischemic mice featured a decrease in the Bacteroidetes and Firmicutes relative abundances in ischemic compared to nonischemic control mice. In this experimental model, baicalin treatment restored the levels of Firmicutes and Bacteroidetes, and reduced TMA and TMAO synthesis without altering the levels of liver FMO3 protein and the activity of hepatic FMO. Also, the effects of baicalin were diminished when ischemic mice were pretreated with broad-spectrum antibiotics to deplete gut microbial populations, suggesting the involvement of gut microbes in the neuroprotective activity of baicalin.

Taking collectively the studies mentioned above, it seems reasonable to suggest that oral administration of food ingredients, including certain dietary fibers, animal and seed oils, and phytochemicals such as phenolic compounds, carotenoids, and other constituents naturally present in plant-based foods may influence the levels of circulating TMAO in animal models and counteract some of its deleterious effects. To note, a number of structurally diverse compounds or extracts seem to affect hepatic FMO activity which may also explain the observed changes in circulating TMAO levels. In spite of the great effort made in the available studies, only very few of them have successfully demonstrated a possible involvement of lower TMAO levels and gut microbiota as part of the underlying mechanism of the observed attenuating effects of the bioactive compounds under study. Some of the reported changes in microbiota by administration of bioactive food compounds affect community structure and composition. As discussed above, some dietary interventions were able to reduce the abundance of microorganisms that can promote TMAO generation, providing a plausible explanation of the observed decrease in TMAO levels. Although various hypotheses can be envisaged to explain such changes in the gut microbial ecology, the mechanistic insights of such community-induced changes have not been thoroughly explored yet. With regard to the link between microbiota and TMAO levels, the potential effects of bioactive food compounds in microbiota metabolism and function is another aspect that remains scarcely investigated. Considering the recent studies reporting on the inhibitory activity of naturally occurring compounds in food against microbial choline TMA-lyase, it can be expected further research aimed at exploring new food sources to attenuate gut microbial TMA generation.

\subsection{Evidence from human studies}

Evidence from human studies is required to better understand the role of the impact of certain dietary components on the composition and metabolism of gut microbiota for CVD prevention. As reported in previous sections, various human intervention studies reporting the effects of TMA precursorsenriched foods or diets on circulating TMAO levels have been published (summarized in Table 1). However, a very limited number of studies have reported the circulating TMAO-lower- ing effects of specific foods, individual food constituents and natural extracts supplementations in humans.

Several of the published studies in humans have detected unexpected changes in TMAO levels in urine as part of the observed data in metabolomic studies. For instance, untargeted metabolomics studies have revealed changes in urinary TMAO levels after interventions with Origanum dictamus tea and Curcuma longa extract as supplements in humans. ${ }^{154,155}$ Although effects on microbiota composition or function was a potential explanation for the observed decrease and increase in urinary TMAO after respective interventions, a possible alteration of kidney function was not excluded. An article by Hernández-Alonso et al. (2017) reported the results of a randomized, controlled, crossover trial with two 4-month dietary intervention arms separated by 2 -week washout period conducted in prediabetic subjects. ${ }^{156}$ During the intervention, two diets were provided, the control diet and a diet supplemented with $57 \mathrm{~g} \mathrm{day}^{-1}$ of pistachio. In the study, $24 \mathrm{~h}$ urine samples, collected at baseline and at the end of each dietary intervention period, were subjected to NMR-based untargeted metabolomic analysis. Interestingly, although pistachios are rich in choline, comparison of the detected urinary metabolites before and after pistachio supplementation revealed that TMAO levels were significantly decreased following pistachio supplemented diet. Also, pistachio supplementation decreased the level dimethylamine compared to that found in the control diet. Other interesting findings in that study were the observed reduction of other two microbial metabolites, namely hippurate and $p$-cresol sulfate, in the urine from subjects after supplementation with pistachio compared to those under control diet.

Besides its role in CVD, TMAO is considered a uremic toxin, and it usually reaches maximum circulating (and urine) levels in individuals with CKD. A report by Hill et al. (2019) explored the feasibility of consuming a high $\beta$-glucan oat supplement and its effect after 12 weeks on uremic toxins and markers of mineral metabolism in CKD patients. ${ }^{157}$ Almost one third of the enrolled patients did not tolerate the $\beta$-glucan oat supplement and withdrew from the study. Serum TMAO level decreased by $17 \%$, and four weeks after discontinuation of the $\beta$-glucan oat supplement, TMAO levels were raised. However, the reduction in TMAO levels was statistically significant when only those patients who had TMAO levels above average healthy levels were included in the analysis, which could be indicative that $\beta$-glucan exerts its greatest effects in CKD patients with high baseline TMAO concentrations. Obeid et al. (2016) reported a study aimed at investigating the effects of dietary supplements other than TMA precursors on plasma TMAO levels in humans. ${ }^{158}$ Their study was based on a randomized single-blinded non-placebo-controlled design in which volunteers without prevalent obesity and diabetes were supplemented with either vitamin D and calcium, or vitamin D, calcium, folic acid, and vitamins B5 and B12 for 12 months. Plasma homocysteine, TMAO and choline metabolites were measured at baseline and at the end of the intervention. Although a $67 \%$ reduction of plasma fasting TMAO was observed after vitamins D and B supplementation, authors dis- 
cussed that the change did not exceed the expected biological variations of TMAO.

As exemplified previously, some of the studies, aimed at investigating the effects of individual phenolic compounds or enriched extracts in animal models, recognize the alteration of microbiota composition or function as a putative mechanism to alter the circulating TMAO levels. The rationale behind the claimed suitability of polyphenols to modulate microbiota composition or function stems from the poor bioavailability of these compounds, which varies depending on its chemical structure. This fact makes some phenolic compounds excellent candidates to reach the distal intestine and exert a significant impact on gut microbiota ecology and metabolism. However, an emerging hypothesis proposes that a potential mechanism of action for the TMAO-lowering effect of certain polyphenols could be based on the ability of such phenolic compounds to donate electrons to TMAO, which acts as an electron acceptor, resulting in reducing TMAO and generation of TMA. It has been speculated that such chemical events could occur at blood level, provided that polyphenols reach blood at physiological relevant levels. These ideas have been proposed in a series of papers reporting randomized trials to study polyphenol-enriched matrices with enhanced characteristics for bioaccessibility of phenolic compounds. ${ }^{159-161}$ In the first study, a microencapsulated grape pomace polyphenolic extract with maltodextrin in acid-resistant capsule registered as Taurisolo® was evaluated in terms of the intestinal bioaccessibility of resveratrol, one of the representative polyphenol in the extract. ${ }^{159}$ The efficacy of the supplement was also assayed in a randomized, placebo-controlled, crossover clinical trial on healthy subjects. More precisely, after a 7-day washout period, volunteers were subjected to a 28 -day intervention period in which capsules containing $300 \mathrm{mg}$ Taurisolo ${ }^{\circledR}$ were administered twice daily. Results indicated that supplementation with grape pomace polyphenolic extract capsules had a lowering effect on TMAO serum levels at the end of the intervention of about $-63.5 \%$. In addition, the reported values of resveratrol in blood after oral administration suggested that the polyphenol was absorbed at the intestinal level and reach the bloodstream. In a subsequent study, authors carried out a monocentric, double-blind, randomized, placebo-controlled, 2-arm parallel-group trial. ${ }^{160}$ In this case, the cohort was constituted of overweight/obese subjects that were subjected to a run-in period lasted 4 weeks, whereas the intervention period and a follow-up period lasted 8 and 4 weeks, respectively. Two intervention groups were established, one supplemented with $300 \mathrm{mg}$ Taurisolo® twice daily and the other supplemented with same dose of Taurisolo $囚$ and $300 \mathrm{mg}$ pectin twice daily. Authors found TMAO serum levels significantly decreased after both interventions, but no significant differences were found between the two interventions, suggesting that pectin does not alter the effect exerted by grape pomace polyphenols. To note, authors discussed that the observed reduction of oxLDL (a circulating oxidative stress biomarker) after polyphenol supplementation reinforces the hypothesis of an antioxidant effect of the supplement exerted at blood level. Despite these relevant observations, the potential effects of the extract on gut microbiota are lacking and mechanistic studies remain to be conducted to confirm the exact underlying mode of action by which Taurisolo ${ }^{\circledR}$ polyphenols would reduce TMAO to TMA.

In a separate report, the effect of lactic fermentation on the bioaccessibility of polyphenols in Annurca apple (an apple variety with high oligomeric procyanidins) was evaluated. ${ }^{161}$ To test its bioactivity, a comparative study of the effects of lactofermented apple puree (inoculated with Lactobacillus rahmnosus and fermented for $24 \mathrm{~h}$ ), the unfermented puree counterpart and the probiotic alone (L. rahmnosus), on plasma lipid profile and TMAO was performed. To achieve this, individuals with CVD risk factors were randomized to three arms, according to a four-weeks run-in period, an eight-weeks intervention, and a four-weeks follow up study. Results indicated that fermentation increased individual free polyphenols from a minimum of $20 \%$ up to $45 \%$. Also, intervention with the lactofermented puree exerted a higher variation of serum parameters, including the elevation of HDL cholesterol by $+61.8 \%$ and the reduction in TMAO levels by $-63.1 \%$. In addition, the three interventions induced a strong increase in Bifidobacterium and Lactobacillus population. Interestingly, unfermented puree supplementation determined the greatest effects on these genera, followed by the intervention with L. rhamnosus, whereas lactofermented puree supplementation induced a significantly minor influence, making reasonable the hypothesis that the supplement richer in intact pectic compounds (unfermented puree) would provide with more substances of prebiotic interest which are well-known to stimulate the growth of fermentative bacteria.

Apart from these encouraging results about the potential of food ingredients and natural extracts to reduce circulating TMAO levels, some other articles can be found in the scientific literature reporting ineffective dietary interventions in humans or interventions that have a TMAO-elevating effect. For instance, Angiletta et al. (2018) evaluated the potential for commonly consumed dietary polyphenols administered at nutritionally relevant doses to reduce TMAO and $\gamma$-butyrobetaine in obese subjects, a population with generally high TMAO levels. ${ }^{162}$ Authors performed a secondary analysis on samples from a previous human clinical study on the impacts of green tea and cocoa supplementation for a period of five days. As the study guidelines involved controlled feeding, it was assumed that the intake of TMAO precursors choline, L-carnitine and betaine were consistent. On the one hand, no significant differences were detected in circulating levels of TMAO and $\gamma$-butyrobetaine across the interventions. On the other hand, authors found great heterogeneity in the intra-individual variability of TMAO concentrations between subjects and interventions. Interestingly, a closer look into the data allowed the identification of "responders" and "non-responders" to the green tea and cocoa beverage with high-flavonoid content; however, no quantitative characteristics, including BMI, body mass, age, fat mass or lean mass were significantly different between responders and non-responders. In this article, authors raised many interesting questions such as the reliability of plasma TMAO measured at a single time point as 
biomarker, and the importance of considering the factors that might influence TMAO variability, including gut microbiota composition, hepatic FMO3 expression and activity, dietary compliance, etc. Baugh et al. (2018) reported another non efficacious dietary intervention intended to reduce circulating TMAO levels. ${ }^{163}$ In this case, a randomized clinical trial to evaluate inulin supplementation in controlled feeding for 6 weeks was proven to be ineffective in reducing either fasting or postprandial TMA and TMAO levels in overweight/obese adults at risk for T2D compared to those detected in placebo group.

\section{Conclusions and future prospects}

It has been demonstrated that diet plays a key role in the production of TMAO since it provides with the nutritional precursors to produce TMA and TMAO. In general, there is association in the levels of circulating TMAO after the consumption of foods rich in dietary trimethylamines; however, some results are conflicting, and therefore, it would be necessary to address, for instance, the different bioavailability of dietary precursors and their effect on gut microbiota composition and its TMA metabolic capacity. In last years, TMA/TMAO metabolism has emerged as an attractive target for prevention and treatment of cardiovascular disease. Targeting microbiota and host metabolic processes involved in TMA and TMAO generation through diet is an approach that holds promise for future intervention. The ability of specific foods, food constituents and phytochemicals found in herbs to lower the levels of circulating TMAO has been mostly demonstrated in animal models. In very few cases, the association between changes in TMAO levels and gut microbiota has been clarified, and the exact mechanisms underlying the effects of the dietary elements object of study remain to be elucidated. More importantly, studies showing that reducing circulating TMAO levels exerts a positive effect in humans are scarce. On this point, as most of the studies have been conducted in animals, the findings are difficult to translate to humans. Future research on this area should face current challenges in microbial research and others more inherent to the study of TMA/TMAO metabolism, as for instance, the high intra-individual variability of plasma TMAO levels observed in some individuals. With the increasingly growing refinement and affordability of next-generation sequencing and other omics technologies, it is expected a shift from studies focusing on describing microbial community composition to more function-oriented research on gut microbiota. Thus, it can be anticipated that shot-gun metagenomics, meta-transcriptomics, meta-proteomics, metabolomics and bioinformatics will be key to disentangle the complex links among diet, microbial metabolism and host health.

\section{Abbreviations}

AMPK Adenosine monophosphate-activated protein kinase

CD36 Cluster of differentiation 36
CDP-choline Cytidine-5-diphosphate-choline

CKD Chronic kidney disease

CVD Cardiovascular disease

DHA Docosahexaenoic acid

DMB 3,3-Dimethyl-1-butanol

EFSA European Food Safety Authority

EPA Eicosapentaenoic acid

EVOO Extra-virgin olive oil

FMO Flavin-containing monooxygenase

FMT Fecal microbiota transplantation

GPC Glycerophosphocholine

HMGB1 High-mobility group B1

HUVEC Human umbilical vein endothelial cell

IL Inflammatory cytokines interleukin

IOM American Institute of Medicine

LDL Low-density lipoprotein

MD Mediterranean diet

NLRP3 Pyrin domain-containing-3

PC Phosphatidylcholine

PChol Phosphocholine

PERK Protein kinase R-like endoplasmic reticulum kinase

PREDIMED PREvention with MEDiterranean Diet

rDNA Ribosomal RNA

RS Resistant starch

SPM Sphingomyelin

T2D Type-2 diabetes

TF Tissue factor

TLR4 Toll-like receptor 4

TMA Trimethylamine

TMAO Trimethylamine $\mathrm{N}$-oxide

TMAU Trimethylaminuria

TML N6,N6,N6-Trimethyl-L-lysine

\section{Financial support}

This work is jointly supported by research grant AGL201789055-R (Ministerio de Ciencia e Innovación, MICINN). We acknowledge support of the publication fee by the CSIC Open Access Publication Support Initiative through its Unit of Information Resources for Research (URICI).

\section{Conflicts of interest}

There are no conflicts of interest.

\section{References}

1 H. C. Descamps, B. Herrmann, D. Wiredu and C. A. Thaiss, The path toward using microbial metabolites as therapies, EBioMedicine, 2019, 44, 747-754.

2 W. Van Treuren and D. Dodd, Microbial contribution to the human metabolome: implications for health 
and disease, Annu. Rev. Pathol.: Mech. Dis., 2020, 15, 345369.

3 Z. Wang and Y. Zhao, Gut microbiota derived metabolites in cardiovascular health and disease, Protein Cell, 2018, 9, 416-431.

4 I. C. van den Munckhof, A. Kurilshikov, R. ter Horst, N. P. Riksen, L. A. Joosten, A. Zhernakova, J. Fu, S. T. Keating, M. G. Netea, J. de Graaf and J. H. Rutten, Role of gut microbiota in chronic low-grade inflammation as potential driver for atherosclerotic cardiovascular disease: A systematic review of human studies, Obes. Rev., 2018, 19, 1719-1734.

5 Y. Du, X. Li, C. Su, L. Wang, J. Jiang and B. Hong, The human gut microbiome - a new and exciting avenue in cardiovascular drug discovery, Expert Opin. Drug Discovery, 2019, 14, 1037-1052.

6 K. Kasahara and F. E. Rey, The emerging role of gut microbial metabolism on cardiovascular disease, Curr. Opin. Microbiol., 2019, 50, 64-70.

7 W. H. Tang, F. Bäckhed, U. Landmesser and S. L. Hazen, Intestinal microbiota in cardiovascular health and disease: JACC state-of-the-art review, JACC, 2019, 73, 20892105.

8 N. Kazemian, M. Mahmoudi, F. Halperin, J. C. Wu and S. Pakpour, Gut microbiota and cardiovascular disease: opportunities and challenges, Microbiome, 2020, 8, 36.

9 M. Onyszkiewicz, K. Jaworska and M. Ufnal, Short chain fatty acids and methylamines produced by gut microbiota as mediators and markers in the circulatory system, Exp. Biol. Med., 2020, 245, 166-175.

10 M. V. Warmbrunn, H. Herrema, J. Aron-Wisnewsky, M. R. Soeters, D. H. Van Raalte and M. Nieuwdorp, Gut microbiota: A promising target against cardiometabolic diseases, Expert Rev. Endocrinol. Metab., 2020, 15, 13-27.

11 H. Xu, X. Wang, W. Feng, Q. Liu, S. Zhou, Q. Liu and L. Cai, The gut microbiota and its interactions with cardiovascular disease, Microb. Biotechnol., 2020, 13, 637656.

12 I. Nemet, P. P. Saha, N. Gupta, W. Zhu, K. A. Romano, S. M. Skye, T. Cajka, M. L. Mohan, L. Li, Y. Wu, M. Fuanbashi, A. E. Ramer-Tait, S. V. Prasad, O. Fiehn, F. E. Rey, W. H. Tang, M. A. Fischbach, J. A. DiDonato and S. L. Hazen, A cardiovascular disease-linked gut microbial metabolite acts via adrenergic receptors, Cell, 2020, 180, 862-877.

13 Z. Wang, E. Klipfell, B. J. Bennett, R. Koeth, B. S. Levison, B. Dugar, A. E. Feldstein, E. B. Britt, X. Fu, Y. M. Chung, Y. Wu, P. Schauer, J. D. Smith, H. Allayee, W. H. Tang, J. A. DiDonato, A. J. Lusis and S. L. Hazen, Gut flora metabolism of phosphatidylcholine promotes cardiovascular disease, Nature, 2011, 472, 57-63.

14 R. A. Koeth, Z. Wang, B. S. Levison, J. A. Buffa, E. Org, B. T. Sheehy, E. B. Britt, X. Fu, Y. Wu, L. Li, J. D. Smith, J. A. DiDonato, J. Chen, H. Li, G. D. Wu, J. D. Lewis, M. Warrier, J. M. Brown, R. M. Krauss, W. H. Tang, F. D. Bushman, A. J. Lusis and S. L. Hazen, Intestinal microbiota metabolism of L-carnitine, a nutrient in red meat, promotes atherosclerosis, Nat. Med., 2013, 19, 576585.

15 R. A. Koeth, B. S. Levison, M. K. Culley, J. A. Buffa, Z. Wang, J. C. Gregory, E. Org, Y. Wu, L. Li, J. D. Smith, W. H. W. Tang, J. A. DiDonato, A. J. Lusis and S. L. Hazen, $\gamma$-Butyrobetaine is a proatherogenic intermediate in gut microbial metabolism of l-carnitine to TMAO, Cell Metab., 2014, 20, 799-812.

16 C. Kanitsoraphan, P. Rattanawong, S. Charoensri and V. Senthong, Trimethylamine N-Oxide and risk of cardiovascular disease and mortality, Curr. Nutr. Rep., 2018, 7, 207-213.

17 S. Yang, X. Li, F. Yang, R. Zhao, X. Pan, J. Liang, L. Tian, X. Li, L. Liu, Y. Xing and M. Wu, Gut microbiota-dependent marker TMAO in promoting cardiovascular disease: inflammation mechanism, clinical prognostic, and potential as a therapeutic target, Front. Pharmacol., 2019, 10, 1360.

18 Y. Heianza, W. Ma, J. E. Manson, K. M. Rexrode and L. Qi, Gut microbiota metabolites and risk of major adverse cardiovascular disease events and death: A systematic review and meta-analysis of prospective studies, J. Am. Heart Assoc., 2017, 6, e004947.

19 A. Nowinski and J. Ufnal, Nutrition, 2018, 46, 7-12.

20 J. Qi, T. You, J. Li, T. Pan, L. Xiang, Y. Han and L. Zhu, Circulating trimethylamine N-oxide and the risk of cardiovascular diseases: A systematic review and meta-analysis of 11 prospective cohort studies, J. Cell. Mol. Med., 2018, 22, 185-194.

21 B. A. Kappel and M. Federici, Gut microbiome and cardiometabolic risk, Rev. Endocr. Metab. Disord., 2019, 20, 399406.

22 T. Katsimichas, A. S. Antonopoulos, A. Katsimichas, T. Ohtani, Y. Sakata and D. Tousoulis, The intestinal microbiota and cardiovascular disease, Cardiovasc. Res., 2019, 115, 1471-1486.

23 X. Sun, X. Jiao, Y. Ma, Y. Liu, L. Zhang, Y. He and Y. Chen, Trimethylamine N-oxide induces inflammation and endothelial dysfunction in human umbilical vein endothelial cells via activating ROS-TXNIP-NLRP3 inflammasome, Biochem. Biophys. Res. Commun., 2016, 481, 63-70.

24 M. L. Chen, X. H. Zhu, L. Ran, H. D. Lan, L. Yi and M. T. Mi, Trimethylamine-N-Oxide induces vascular inflammation by activating the nlrp3 inflammasome through the sirt3-sod2-mtros signaling pathway, J. Am. Heart Assoc., 2017, 6, e006347.

25 Y. Ke, D. Li, M. Zhao, C. Liu, J. Liu, A. Zeng, X. Shi, S. Chen, B. Pan, L. Zheng and H. Hong, Gut flora-dependent metabolite trimethylamine-N-oxide accelerates endothelial cell senescence and vascular aging through oxidative stress, Free Radical Biol. Med., 2018, 116, 88-100.

26 G. Ma, B. Pan, Y. Chen, C. Guo, M. Zhao, L. Zheng and B. Chen, Trimethylamine N-oxide in Atherogenesis: Impairing Endothelial Self-Repair Capacity and Enhancing Monocyte Adhesion, Biosci. Rep., 2017, 37, BSR20160244. 
27 M. M. Seldin, Y. Meng, H. Qi, W. Zhu, Z. Wang, S. L. Hazen, A. J. Lusis and D. M. Shih, Trimethylamine $\mathrm{N}$-Oxide promotes vascular inflammation through signaling of Mitogen-Activated Protein Kinase and Nuclear Factor-кB, J. Am. Heart Assoc., 2016, 5, e002767.

28 X. Zhang, Y. Li, P. Yang, X. Iu, L. Lu, Y. Chen, X. Zhong, Z. Li, H. Liu, C. Ou, J. Yan and M. Chen, Trimethylamine$\mathrm{N}$-Oxide promotes vascular calcification through activation of NLRP3 (Nucleotide-Binding Domain, LeucineRich-Containing Family, Pyrin Domain-Containing-3) Inflammasome and NF- $\mathrm{BB}$ (Nuclear Factor $\kappa \mathrm{B}$ ) Signals, Arterioscler. Thromb. Vasc. Biol., 2020, 40, 751-765.

29 G. B. Singh, Y. Zhang, K. M. Boini and S. Koka, High Mobility Group Box 1 Mediates TMAO-Induced Endothelial Dysfunction, Int. J. Mol. Sci., 2019, 20, 3570.

30 X. Cheng, X. Qiu, Y. Liu, C. Yuan and X. Yang, Trimethylamine N-oxide promotes tissue factor expression and activity in vascular endothelial cells: A new link between Trimethylamine N-oxide and atherosclerotic thrombosis, Thromb. Res., 2019, 177, 110-116.

31 J. Geng, C. Yang, B. Wang, X. Zhang, T. Hu, Y. Gu and J. Li, Trimethylamine N-oxide promotes atherosclerosis via CD36-dependent MAPK/JNK Pathway, Biomed. Pharmacother., 2018, 97, 941-947.

32 W. Zhu, J. C. Gregory, E. Org, J. A. Buffa, N. Gupta, Z. Wang, L. Li, X. Fu, Y. Wu, M. Mehrabian, R. B. Sartor, T. M. McIntyre, R. L. Silverstein, W. H. Tang, J. A. Didonato, J. M. Brown, A. J. Lusis and S. L. Hazen, Gut microbial metabolite TMAO enhances platelet hyperreactivity and thrombosis risk, Cell, 2016, 165, 111124.

33 S. Subramaniam, S. Boukhlouf and C. Fletcher, A bacterial metabolite, trimethylamine $\mathrm{N}$-oxide, disrupts the hemostasis balance in human primary endothelial cells but no coagulopathy in mice, Blood Coagulation Fibrinolysis, 2019, 30, 324-330.

34 S. Chen, A. Henderson, M. C. Petriello, K. A. Romano, M. Gearing, J. Miao, M. Schell, W. J. Sandoval-Espinola, J. Tao, B. Sha, M. Grahan, R. Crooke, A. Kleinridders, E. P. Balsjus, F. E. Rey, A. J. Morris and S. B. Biddinger, Trimethylamine N-Oxide binds and activates PERK to promote metabolic dysfunction, Cell Metab., 2019, 30, 1141-1151.

35 C. Lebeaupin, E. Proics, C. H. de Bieville, D. Rousseau, S. Bonnafous, S. Patouraux, G. Adam, V. J. Lavallard, C. Rovere, O. Le Thuc, M. C. Saint-Paul, R. Anti, A. S. Schneck, A. Lannelli, J. Gugenheim, A. Tran, P. Gual and B. Bally-Maitre, ER stress induces NLRP3 inflammasome activation and hepatocyte death, Cell Death Dis., 2015, 6, e1879.

36 A. B. Tam, E. L. Mercado, A. Hoffmann and M. Niwa, ER stress activates NF- $\kappa$ B by integrating functions of basal IKK activity, IRE1 and PERK, PLoS One, 2012, 7, e45078.

37 S. H. Liang, W. Zhang, B. C. McGrath, P. Zhang and D. R. Cavener, PERK (eIF2 $\alpha$ kinase) is required to activate the stress-activated MAPKs and induce the expression of immediate-early genes upon disruption of ER calcium homoeostasis, Biochem. J., 2006, 393, 201-209.

38 S. Cracium and E. P. Balskus, Microbial conversion of choline to trimethylamine requires a glycyl radical enzyme, Proc. Natl. Acad. Sci. U. S. A., 2012, 109, 2130721312.

39 Y. Zhu, E. Jameson, M. Crosatti, H. Schäfer, K. Rajakumar, T. D. Bugg and Y. Chen, carnitine metabolism to trimethylamine by an unusual Rieske-type Oxygenase from human microbiota, Proc. Natl. Acad. Sci. U. S. A., 2014, 111, 4268-4273.

40 G. Falony, S. Vieira-Silva and J. Raes, Microbiology meets big data: The case of gut microbiota-derived trimethylamine, Annu. Rev. Microbiol., 2015, 69, 305-321.

41 Z. Wang, A. B. Roberts, J. A. Buffa, B. S. Levison, W. Zhu, E. Org, X. Gu, Y. Huang, M. Zamanian-Daryoush, M. K. Culley, A. J. DiDonato, X. Fu, J. E. Hazen, D. Krajcik, J. A. DiDonato, A. J. Lusis and S. L. Hazen, Non-lethal inhibition of gut microbial trimethylamine production for the treatment of atherosclerosis, Cell, 2015, 163, 15851595.

42 J. R. Andreessen, Glycine metabolism in anaerobes, Anton. Leeuw. Int. J. G., 1994, 66, 223-237.

43 S. L. McCrindle, U. Kappler and A. G. McEwan, Microbial dimethylsulfoxide and trimethylamine-N-oxide respiration, Adv. Microb. Physiol., 2005, 50, 147-197.

44 K. A. Romano, E. I. Vivas, D. Amador-Noguez and F. E. Rey, Intestinal microbiota composition modulates choline bioavailability from diet and accumulation of the proatherogenic metabolite trimethylamine-N-oxide, mBio, 2015, 6, 1-8.

45 E. Jameson, A. C. Doxey, R. Airs, K. J. Purdy, J. C. Murrell and Y. Chen, Metagenomic data-mining reveals contrasting microbial populations responsible for trimethylamine formation in human gut and marine ecosystems, Microb. Genomes, 2016, 2, e000080.

46 L. Gram and P. Dalgaard, Curr. Opin. Biotechnol., 2002, 13, 262-266.

47 C. E. Hebard, G. J. Flick and R. E. Martin, Occurrence and significance of trimethylamine oxide and its derivatives in fish and shellfish, in Chemistry and Biochemistry of Marine Food Products, ed. R. E. Martin, G. J. Flick, C. E. Hebard and D. R. Ward, AVI Publishing Company, Westport, CT, 1982, pp. 149-304.

48 G. Summers, R. D. Wibisono, D. I. Hedderley and G. C. Fletcher, Trimethylamine oxide content and spoilage potential of New Zealand commercial fish species, N. Z. J. Mar. Freshwater Res., 2017, 51, 393-405.

49 A. Steiber, J. Kerner and C. L. Hoppel, Carnitine: A Nutritional, biosynthetic, and functional perspective, Mol. Aspects Med., 2004, 25, 455-473.

50 S. Knüttel-Gustavsen and J. Harmeyer, The determination of L-carnitine in several food samples, Food Chem., 2007, 105, 793-804.

51 European Food Safety Authority, Dietary reference values for choline, EFSA J., 2016, 14, e04484. 
52 Institute of Medicine (IOM), Dietary Reference Intakes for Thiamin, Riboflavin, Niacin, Vitamin B6, Folate, Vitamin B12, Pantothenic Acid, Biotin, and Choline, The National Academy Press, Washington, DC, USA, 1998.

53 K. Patterson, S. Bhagwat, J. Williams, J. Howe, J. Holden, S. Zeisel, K. Dacosta and M. Mar, USDA Database for the Choline Content of Common Foods, release two, U.S. Department of Agriculture, 2008.

54 C. Böttcher, E. von Roepenack-Lahaye, J. Schmidt, S. Clemens and D. Scheel, Analysis of phenolic choline esters from seeds of Arabidopsis thaliana and Brassica napus by capillary liquid chromatography/electrospraytandem mass spectrometry, J. Mass Spectrom., 2009, 44, 466-476.

55 H. Chen, L. Peng, M. Pérez de Nanclares, M. P. Trudeau, D. Yao, Z. Cheng, P. E. Urriola, L. T. Mydland, G. C. Shurson, M. Overland and C. Chen, Identification of sinapine-derived choline from a rapeseed diet as a source of serum trimethylamine N-oxide in pigs, J. Agric. Food Chem., 2019, 67, 7748-7754.

56 L. Servillo, A. Giovane, D. Cautela, D. Castaldo and M. L. Balestrieri, Where does $\mathrm{N}(\varepsilon)$-trimethyllysine for the carnitine biosynthesis in mammals come from?, PLoS One, 2014, 9, e84589.

57 X. S. Li, Z. Wang, T. Cajka, J. A. Buffa, I. Nemet, A. G. Hurd, X. Gu, S. M. Skye, A. B. Roberts, Y. Wu, L. Li, C. J. Shahen, M. A. Wagner, J. A. Hartiala, R. L. Kerby, K. A. Romano, Y. Han, S. Obeid, T. F. Lüscher, H. Allayee, F. E. Rey, J. A. DiDonato, O. Fiehn, W. H. W. Tang and S. L. Hazen, Untargeted metabolomics identifies trimethyllysine, a TMAO-producing nutrient precursor, as a predictor of incident cardiovascular disease risk, JCI Insight, 2018, 3, 99096.

58 B. Filipčev, J. Kojić, J. Krulj, M. Bodroža-Solarov and N. Ilić, Betaine in cereal grains and grain-based products, Foods, 2018, 7, E49.

59 S. H. Zeisel, M.-H. Mar, J. C. Howe and J. M. Holden, Concentrations of choline-containing compounds and betaine in common foods, J. Nutr., 2003, 133, 13021307.

60 J. Ey, E. Schömig and D. Taubert, Dietary sources and antioxidant effects of ergothioneine, J. Agric. Food Chem., 2007, 55, 6466-6474.

61 L. Servillo, N. D'Onofrio, A. Giovane, R. Casale, D. Cautela, D. Castaldo, F. Iannaccone, G. Neglia, G. Campanile and M. L. Balestrieri, Ruminant Meat and milk contain $\delta$-valerobetaine, another precursor of trimethylamine N-oxide (TMAO) like $\gamma$-butyrobetaine, Food Chem., 2018, 260, 193-199.

62 J. Hartiala, B. J. Bennett, W. H. Tang, Z. Wang, A. F. Stewart, R. Roberts, R. McPherson, A. J. Lusis, S. L. Hazen and H. Allayee, Comparative genome-wide association studies in mice and humans for trimethylamine N-Oxide, a proatherogenic metabolite of choline and L-carnitine, Arterioscler. Thromb. Vasc. Biol., 2014, 34, 1307-1313.
63 M. E. Dumas, E. C. Maibaum, C. Teague, H. Ueshima, B. Zhou, J. C. Lindon, J. K. Nicholson, J. Stamler, P. Elliott, Q. Chan and E. Holmes, Assessment of analytical reproducibility of ${ }^{1} \mathrm{H}$ NMR spectroscopy based metabonomics for large-scale epidemiological research: The INTERMAP Study, Anal. Chem., 2006, 78, 2199-2208.

64 R. Gibson, C.-H. E. Lau, R. L. Loo, T. M. D. Ebbels, E. Chekmeneva, A. R. Dyer, K. Miura, H. Ueshima, L. Zhao, M. L. Daviglus, J. Stamler, L. Van Horn, P. Elliott, E. Holmes and Q. Chan, The association of fish consumption and its urinary metabolites with cardiovascular risk factors: The International Study of Macro-/Micronutrients and Blood Pressure (INTERMAP), Am. J. Clin. Nutr., 2020, 111, 280-290.

65 R. Krüger, B. Merz, M. J. Rist, P. G. Ferrario, A. Bub, S. E. Kulling and B. Watzl, Associations of current diet with plasma and urine TMAO in the KarMeN Study: Direct and indirect contributions, Mol. Nutr. Food Res., 2017, 61, 1700363.

66 A. J. Lloyd, G. Favé, M. Beckmann, W. Lin, K. Tailliart, L. Xie, J. C. Mathers and J. Draper, Use of mass spectrometry fingerprinting to identify urinary metabolites after consumption of specific foods, Am. J. Clin. Nutr., 2011, 94, 981-991.

67 M. Dambrova, E. Skapare-makarova, I. Konrade, O. Pugovics, S. Grinberga, D. Tirzite, R. Petrovska, I. Kalvins and E. Liepins, Meldonium decreases the dietincreased plasma levels of trimethylamine N-oxide, a metabolite associated with atherosclerosis, J. Clin. Pharmacol., 2013, 53, 1095-1098.

68 W. Cheung, P. Keski-Rahkonen, N. A. Assi, P. Ferrari, H. Freisling, S. Rinaldi, N. Slimani, R. Zamora-Ros, M. Rundle, G. Frost, H. Gibbons, E. Carr, L. Brennan, A. J. Cross, V. Pala, S. Panico, C. Sacerdote, D. Palli, R. Tumino, T. Kühn, R. Kaaks, H. Boeing, A. Floegel, F. Mancini, M.-C. Boutron-Ruault, L. Baglietto, A. Trichopoulou, A. Naska, P. Orfanos and A. Scalbert, A metabolomic study of biomarkers of meat and fish intake, Am. J. Clin. Nutr., 2017, 105, 600-608.

69 C. E. Cho, S. Taesuwan, O. V. Malysheva, E. Bender, N. F. Tulchinsky, J. Yan, J. L. Sutter and M. A. Caudill, Trimethylamine-N-oxide (TMAO) response to animal source foods varies among healthy young men and is influenced by their gut microbiota composition: A randomized controlled trial, Mol. Nutr. Food Res., 2016, 1-12.

70 X. Yin, H. Gibbons, M. Rundle, G. Frost, B. A. McNulty, A. P. Nugent, J. Walton, A. Flynn and L. Brennan, The relationship between fish intake and urinary trimethylamine-N-Oxide, Mol. Nutr. Food Res., 2020, 64, 1900799.

71 W. H. W. Tang, Z. Wang, B. S. Levison, R. A. Koeth, E. B. Britt, X. Fu, Y. Wu and S. L. Hazen, Intestinal microbial metabolism of phosphatidylcholine and cardiovascular risk, N. Engl. J. Med., 2013, 368, 1575-1584.

72 C. A. Miller, K. D. Corbin, K. A. da Costa, S. Zhang, X. Zhao, J. A. Galanko, T. Blevins, J. Bennett, A. O'Connor and S. H. Zeisel, Effect of egg ingestion on trimethyl- 
amine-n-oxide production in humans: A randomized, controlled, dose-response study, Am. J. Clin. Nutr., 2014, 100, 778-786.

73 A. A. West, Y. Shih, W. Wang, K. Oda, K. Jaceldo-Siegl, J. Sabaté, E. Haddad, S. Rajaram, M. A. Caudill and B. Burns-Whitmore, Egg n-3 Fatty Acid Composition Modulates Biomarkers of Choline Metabolism in FreeLiving Lacto-Ovo-Vegetarian Women of Reproductive Age, J. Acad. Nutr. Diet., 2014, 114, 1594-1600.

74 D. M. DiMarco, A. Missimer, A. G. Murillo, B. S. Lemos, O. V. Malysheva, M. A. Caudill, C. N. Blesso and M. L. Fernandez, Intake of up to 3 eggs/day increases hdl cholesterol and plasma choline while plasma trimethylamine-N-oxide is unchanged in a healthy population, Lipids, 2017, 52, 255-263.

75 B. S. Lemos, I. Medina-Vera, O. V. Malysheva, M. A. Caudill and M. L. Fernandez, Effects of egg consumption and choline supplementation on plasma choline and trimethylamine-n-oxide in a young population, J. Am. Coll. Nutr., 2018, 15, 1-8.

76 A. Missimer, M. L. Fernandez, D. M. DiMarco, G. H. Norris, C. N. Blesso, A. G. Murilloa, M. VergaraJimenez, B. S. Lemos, I. Medina-Vera, O. V. Malysheva and M. A. Caudill, Compared to an oatmeal breakfast, two eggs/day increased plasma carotenoids and choline without increasing trimethyl amine N-Oxide concentrations, J. Am. Coll. Nutr., 2018, 37, 140-148.

77 R. Obeid, H. M. Awwad, M. Keller and J. Geisel, Trimethylamine-N-oxide and its biological variations in vegetarians, Eur. J. Nutr., 2017, 56, 2599-2609.

78 Y. Mödinger, C. Schön, M. Wilhelm and P.-A. Hals, Plasma kinetics of choline and choline metabolites after a single dose of SuperbaBoost ${ }^{\mathrm{TM}}$ krill oil or choline bitartrate in healthy volunteers, Nutrients, 2019, 11, 2548.

79 R. K. Berge, M. S. Ramsvik, P. Bohov, A. Svardal, J. E. Nordrehaug, E. Rostrup, I. Bruheim and B. Bjørndal, Krill oil reduces plasma triacylglycerol level and improves related lipoprotein particle concentration, fatty acid composition and redox status in healthy young adults - A pilot study, Lipids Health Dis., 2015, 14, 163.

80 H.-K. Biesalski, Meat as a component of a healthy diet Are there any risks or benefits if meat is avoided in the diet?, Meat Sci., 2005, 70, 509-524.

81 C. S. Williamson, R. K. Foster, S. A. Stanner and J. L. Buttriss, Red meat in the diet, Nutr. Bull., 2005, 30, 323-355.

82 W. Zhu, Z. Wang, W. H. W. Tang and S. L. Hazen, Gut microbe-generated trimethylamine $\mathrm{N}$-oxide from dietary choline is prothrombotic in subjects, Circulation, 2017, 135, 1671-1673.

83 J. Xu, S. Yang, S. Cai, J. Dong, X. Li and Z. Chen, Identification of Biochemical Changes in Lactovegetarian Urine Using ${ }^{1} \mathrm{H}$ NMR Spectroscopy and Pattern Recognition, Anal. Bioanal. Chem., 2010, 396, 1451-1463.

84 R. A. Koeth, B. R. Lam-Galvez, J. Kirsop, Z. Wang, B. S. Levison, X. Gu, M. F. Copeland, D. Bartlett,
D. B. Cody, H. J. Dai, M. K. Culley, X. S. Li, X. Fu, Y. Wu, L. Li, J. A. DiDonato, W. H. W. Tang, J. C. Garcia-Garcia and S. L. Hazen, L-Carnitine in omnivorous diets induces an atherogenic gut microbial pathway in humans, J. Clin. Invest., 2019, 129, 373-387.

85 C. Stella, B. Beckwith-Hall, O. Cloarec, E. Holmes, J. C. Lindon, J. Powell, F. van der Ouderaa, S. Bingham, A. J. Cross and J. K. Nicholson, Susceptibility of Human Metabolic Phenotypes to Dietary Modulation, J. Proteome Res., 2006, 5(10), 2780-2788.

86 P. Giesbertz, B. Brandl, Y.-M. Lee, H. Hauner, H. Daniel and T. Skurk, Specificity, dose dependency, and kinetics of markers of chicken and beef intake using targeted quantitative LC-MS/MS: A human intervention trial, Mol. Nutr. Food Res., 2020, 64, 1900921.

87 Z. Wang, N. Bergeron, B. S. Levison, X. S. Li, S. Chiu, X. Jia, R. A. Koeth, L. Li, Y. Wu, W. H. W. Tang, R. M. Krauss and S. L. Hazen, Impact of chronic dietary red meat, white meat, or non-meat protein on trimethylamine N-oxide metabolism and renal excretion in healthy men and women, Eur. Heart J., 2019, 40, 583-594.

88 M.-B. S. Andersen, H. C. Reinbach, A. Rinnan, T. Barri, C. Mithril and L. O. Dragsted, Discovery of exposure markers in urine for Brassica-containing meals served with different protein sources by UPLC-qTOF-MS untargeted metabolomics, Metabolomics, 2013, 9, 984-997.

89 T. Kühn, S. Rohrmann, D. Sookthai, T. Johnson, V. Katzke, R. Kaaks, A. Von Eckardstein and D. Müller, Intra-individual variation of plasma trimethylamine-N-oxide (TMAO), betaine, and choline over 1 year, Clin. Chem. Lab. Med., 2016, 55, 264-268.

90 S. Rohrmann, J. Linseisen, M. Allenspach, A. von Eckardstein and D. Muüller, Plasma concentrations of trimethylamine-N-oxide are directly associated with dairy food consumption and low-grade inflammation in a german adult population, J. Nutr., 2016, 146, 283-289.

91 K. J. Burton, R. Krüger, V. Scherz, L. H. Münger, G. Picone, N. Vionnet, C. Bertelli, G. Greub, F. Capozzi and G. Vergères, Trimethylamine- N-oxide postprandial response in plasma and urine is lower after fermented compared to non-fermented dairy consumption in healthy adults, Nutrients, 2020, 12, 234.

92 M. Schmedes, A. D. Brejnrod, E. K. Aadland, P. Kiilerich, K. Kristiansen, H. Jacques, C. Lavigne, I. E. Graff, Ø. Eng, A. Holthe, G. Mellgren, J. F. Young, U. K. Sundekilde, B. Liaset and H. C. Bertram, Lean-seafood intake decreases urinary markers of mitochondrial lipid and energy metabolism in healthy subjects: Metabolomics results from a randomized crossover intervention study, Mol. Nutr. Food Res., 2019, 63, e1700976.

93 N. Bergeron, P. T. Williams, R. Lamendella, N. Faghihnia, A. Grube, X. Li, Z. Wang, R. Knight, J. K. Jansson, S. L. Hazen and R. M. Krauss, Diets high in resistant starch increase plasma levels of trimethylamine-N-oxide, a gut microbiome metabolite associated with cvd risk, Br. J. Nutr., 2016, 116, 2020-2029. 
94 G. D. Wu, J. Chen, C. Hoffmann, K. Bittinger, Y. Y. Chen, S. A. Keilbaugh, M. Bewtra, D. Knights, W. A. Walters, R. Knight, R. Sinha, E. Gilroy, K. Gupta, R. Baldassano, L. Nessel, H. Li, F. D. Bushman and J. D. Lewis, Linking long-term dietary patterns with gut microbial enterotypes, Science, 2011, 334, 105-108.

95 N. E. Boutagy, A. P. Neilson, K. L. Osterberg, A. T. Smithson, T. R. Englund, B. M. Davy, M. W. Hulver and K. P. Davy, Short-term high-fat diet increases postprandial trimethylamine-N-oxide in humans, Nutr. Res., 2015, 35, 858-864.

96 A. M. Malinowska, A. Szwengiel and A. Chmurzynsk, Dietary, anthropometric, and biochemical factors influencing plasma choline, carnitine, trimethylamine, and trimethylamine-N-oxide concentrations, Int. J. Food Sci. Nutr., 2017, 68, 488-495.

97 A. O’Sullivan, M. J. Gibney and L. Brennan, Dietary intake patterns are reflected in metabolomic profiles: potential role in dietary assessment studies, Am. J. Clin. Nutr., 2011, 93, 314-321.

98 L. Serra-Majem, B. Román-Viñas, A. Sanchez-Villegas, M. Guasch-Ferré, D. Corella and C. La Vecchia, Benefits of the mediterranean diet: Epidemiological and molecular aspects, Mol. Aspects Med., 2019, 67, 1-55.

99 W. C. Willett, F. Sacks, A. Trichopoulou, G. Drescher, A. Ferro-Luzzi, E. Helsing and D. Trichopoulos, Mediterranean diet pyramid: A cultural model for healthy eating, Am. J. Clin. Nutr., 1995, 61, 1402S-1406S.

100 A. Bach-Faig, E. M. Berry, D. Lairon, J. Reguant, A. Trichopoulou, S. Dernini, F. X. Medina, M. Battino, R. Belahsen, G. Miranda and L. Serra-Majem, Mediterranean diet pyramid today. Science and cultural updates, Public Health Nutr., 2011, 14, 2274-2284.

101 R. Estruch, E. Ros, J. Salas-Salvadó, M. I. Covas, D. Corella, F. Arós, E. Gómez-Gracia, V. Ruiz-Gutiérrez, M. Fiol, J. Lapetra, R. M. Lamuela-Raventos, L. SerraMajem, X. Pintó, J. Basora, M. A. Muñoz, J. V. Sorlí, J. A. Martínez and M. A. Martínez-González, Primary prevention of cardiovascular disease with a Mediterranean diet, N. Engl. J. Med., 2013, 368, 1279-1290.

102 R. Vázquez-Fresno, R. Llorach, M. Urpi-Sarda, A. Lupianez-Barbero, R. Estruch, D. Corella, M. Fitó, F. Arós, M. Ruiz-Canela, J. Salas-Salvadó and C. AndresLacueva, Metabolomic pattern analysis after mediterranean diet intervention in a nondiabetic population: A1and 3-year follow-up in the PREDIMED Study, J. Proteome Res., 2015, 14, 531-540.

103 M.Á Martínez-González, D. Corella, J. Salas-Salvadó, E. Ros, M. I. Covas, M. Fiol, J. Wärnberg, F. Arós, V. RuízGutiérrez, R. M. Lamuela-Raventós, J. Lapetra, M. Á Muñoz, J. A. Martínez, G. Sáez, L. Serra-Majem, X. Pintó, M. T. Mitjavila, J. A. Tur, M. P. Portillo and R. Estruch, Cohort Profile: Design and methods of the PREDIMED Study, Int. J. Epidemiol., 2012, 41, 377-385.

104 E. Almanza-Aguilera, M. Urpi-Sarda, R. Llorach, R. Vázquez-Fresno, M. Garcia-Aloy, F. Carmona,
A. Sanchez, F. Madrid-Gambin, R. Estruch, D. Corella and C. Andres-Lacueva, Microbial metabolites are associated with a high adherence to a mediterranean dietary pattern using a 1 h-nmr-based untargeted metabolomics approach, J. Nutr. Biochem., 2017, 48, 36-43.

105 M. A. Martínez-González, A. García-Arellano, E. Toledo, J. Salas-Salvadó, P. Buil-Cosiales, D. Corella, M. I. Covas, H. Schröder, F. Arós, E. Gómez-Gracia, M. Fiol, V. RuizGutiérrez, J. Lapetra, R. M. Lamuela-Raventos, L. SerraMajem, X. Pintó, M. A. Muñoz, J. Wärnberg, E. Ros and R. Estruch, A 14-item Mediterranean diet assessment tool and obesity indexes among high-risk subjects: The PREDIMED Trial, PLoS One, 2012, 7, e43134.

106 L. Barrea, G. Annunziata, G. Muscogiuri, D. Laudisio, C. Di Somma, M. Maisto, G. C. Tenore, A. Colao and S. Savastano, Trimethylamine N-oxide, Mediterranean diet, and nutrition in healthy, normal-weight adults: also a matter of sex?, Nutrition, 2019, 62, 7-17.

107 F. De Filippis, N. Pellegrini, L. Vannini, I. B. Jeffery, A. L. Storia, L. Laghi, D. I. Serrazanetti, R. D. Cagno, I. Ferrocino, C. Lazzi, S. Turroni, L. Cocolin, P. Brigidi, E. Neviani, M. Gobbetti, P. W. O'Toole and D. Ercolini, High-level adherence to a Mediterranean diet beneficially impacts the gut microbiota and associated metabolome, Gut, 2016, 65, 1812-1821.

108 L. E. Griffin, Z. Djuric, C. J. Angiletta, C. M. Mitchell, M. E. Baugh, K. P. Davy and A. P. Neilson, A Mediterranean diet does not alter plasma trimethylamine $\mathrm{N}$-oxide concentrations in healthy adults at risk for colon cancer, Food Funct., 2019, 10, 2138-2147.

109 M. Pignanelli, C. Just, C. Bogiatzi, V. Dinculescu, G. B. Gloor, E. Allen-Vercoe, G. Reid, B. L. Urquhart, K. N. Ruetz, T. J. Velenosi and J. D. Spence, Mediterranean Diet Score: Associations with metabolic products of the intestinal microbiome, carotid plaque burden, and renal function, Nutrients, 2018, 10, E779.

110 D. Fennema, I. R. Phillips and E. A. Shepard, Trimethylamine and Trimethylamine N-Oxide, a FlavinContaining Monooxygenase 3 (FMO3)-mediated hostmicrobiome metabolic axis implicated in health and disease, Drug Metab. Dispos., 2016, 44, 1839-1850.

111 J. Miao, A. V. Ling, P. V. Manthena, M. E. Gearing, M. J. Graham, R. M. Crooke, K. J. Croce, R. M. Esquejo and C. B. Clish, Flavin-containing monooxygenase 3 as a potential player in diabetes-associated atherosclerosis, Nat. Commun., 2015, 6, 6498.

112 J. C. Gregory, J. A. Buffa, E. Org, Z. Wang, B. S. Levison, W. Zhu, M. A. Wagner, B. J. Bennett, L. Li, J. A. DiDonato, A. J. Lusis and S. L. Hazen, Transmission of atherosclerosis susceptibility with gut microbial transplantation, J. Biol. Chem., 2015, 290, 5647-5660.

113 S. M. Skye, W. Zhu, K. A. Romano, C. J. Guo, Z. Wang, X. Jia, J. Kirsop, B. Haag, J. M. Lang, J. A. DiDonato, W. H. Tang, A. J. Lusis, F. E. Rey, M. A. Fischbach and S. L. Hazen, Microbial transplantation with human gut commensals containing CutC is sufficient to transmit 
enhanced platelet reactivity and thrombosis potential, Circ. Res., 2018, 123, 1164-1176.

114 L. P. Smits, R. S. Kootte, E. Levin, A. Prodan, S. Fuentes, E. G. Zoetendal, Z. Wang, B. S. Levison, M. C. Cleophas, M. Kemper, G. M. Dallinga-Tie, A. K. Groen, L. A. Joosten, M. G. Netea, E. S. Stroes, W. M. de Vos, S. L. Hazen and M. Nieuwdorp, Effect of vegan fecal microbiota transplantation on carnitine- and choline-derived Trimethylamine$\mathrm{N}$-Oxide production and vascular inflammation in patients with metabolic syndrome, J. Am. Heart Assoc., 2018, 7, e008342.

115 Y. Zhou, H. Xu, H. Huang, Y. Li, H. Chen, J. He, Y. Du, Y. Chen, Y. Zhou and Y. Nie, Are there potential applications of fecal microbiota transplantation beyond intestinal disorders?, BioMed. Res. Int., 2019, 2019, 3469754.

116 A. U. Din, A. Hassan, Y. Zhu, T. Yin, H. Gregersen and G. Wang, Amelioration of TMAO through probiotics and its potential role in atherosclerosis, Appl. Microbiol. Biotechnol., 2019, 103, 9217-9228.

117 N. E. Boutagy, A. P. Neilson, K. L. Osterberg, A. T. Smithson, T. R. Englund, B. M. Davy, M. W. Hulver and K. P. Davy, Probiotic supplementation and trimethylamine-N-oxide production following a high-fat diet, Obesity, 2015, 23, 2357-2363.

118 N. J. Tripolt, B. Leber, A. Triebl, H. Köfeler, V. Stadlbauer and H. Sourij, Effect of Lactobacillus casei Shirota supplementation on trimethylamine-N-oxide levels in patients with metabolic syndrome: An open-label, randomized study, Atherosclerosis, 2015, 242, 141-144.

119 N. A. Borges, P. Stenvinkel, P. Bergman, A. R. Qureshi, B. Lindholm, C. Moraes, M. B. Stockler-Pinto and D. Madra, Effects of probiotic supplementation on trimethylamine-N-oxide plasma levels in hemodialysis patients: a Pilot Study, Probiotics Antimicrob. Proteins, 2019, 11, 648-654.

120 C. Montruccio, A. De Nicolò, G. D’Ettorre, F. D’Ascenzo, A. Lazzaro, M. Tettoni, A. D'Avolio, S. Bonora, L. Celani, G. Di Perri and A. Calcagno, Serum trimethylamine-Noxide concentrations in people living with hiv and the effect of probiotic supplementation, Int. J. Antimicrob. Agents, 2020, 55, 105908.

121 M. Matsumoto, Y. Kitada, Y. Shimomura and Y. Naito, Bifidobacterium animalis subsp. lactis LKM512 reduces levels of intestinal trimethylamine produced by intestinal microbiota in healthy volunteers: A double-blind, placebocontrolled study, J. Funct. Foods, 2017, 36, 94-101.

122 L. Qiu, D. Yang, X. Tao, J. Yu, H. Xiong and H. Wei, Enterobacter aerogenes ZDY01 attenuates choline-induced trimethylamine N-Oxide levels by remodeling gut microbiota in mice, J. Microbiol. Biotechnol., 2017, 27, 14911499.

123 A. B. Roberts, X. Gu, J. A. Buffa, A. G. Hurd, Z. Wang, W. Zhu, N. Gupta, S. M. Skye, D. B. Cody, B. S. Levison, W. T. Barrington, M. W. Russell, J. M. Reed, A. Duzan, J. M. Lang, X. Fu, L. Li, A. J. Myers, S. Rachakonda, J. A. DiDonato, J. Brown, V. Gogonea, A. J. Lusis,
J. C. Garcia-Garcia and S. L. Hazen, Development of a gut microbe-targeted nonlethal therapeutic to inhibit thrombosis potential, Nat. Med., 2018, 24, 1407-1417.

124 M. Orman, S. Bodea, M. A. Funk, A. Martínez-del Campo, M. Bollenbach, C. L. Drennan and E. P. Balskus, Structure-guided identification of a small molecule that inhibits anaerobic choline metabolism by human gut bacteria, J. Am. Chem. Soc., 2019, 141, 33-37.

125 J. Kuka, E. Liepinsh, M. Makrecka-Kuka, J. Liepins, H. Cirule, D. Gustina, E. Loza, O. Zharkova-Malkova, S. Grinberga, O. Pugovics and M. Dambrova, suppression of intestinal microbiota-dependent production of proatherogenic trimethylamine $\mathrm{N}$-oxide by shifting l-carnitine microbial degradation, Life Sci., 2014, 117, 84-92.

126 L. Bresciani, M. Dall'Asta, C. Favari, L. Calani, D. del Río and F. Brighenti, An in vitro exploratory study of dietary strategies based on polyphenol-rich beverages, fruit juices and oils to control trimethylamine production in the colon, Food Funct., 2018, 9, 6470.

127 X. Gao, X. Liu, J. Xu, C. Xue, Y. Xue and Y. Wang, Dietary Trimethylamine N-oxide exacerbates impaired glucose tolerance in mice fed a high fat diet, J. Biosci. Bioeng., 2014, 118(4), 476-481.

128 X. Gao, J. Xu, C. Jiang, Y. Zhang, Y. Xue, Z. Li, J. Wang, C. Xue and U. Wang, Fish oil ameliorates trimethylamine $\mathrm{N}$-oxide-exacerbated glucose intolerance in high-fat dietfed mice, Food Funct., 2015, 6, 1117.

129 J. Yu, T. Zhang, X. Gao, C. Xue, J. Xu and Y. Wang, Fish oil affects the metabolic process of trimethylamine $\mathrm{N}$-oxide precursor through trimethylamine production and flavincontaining monooxygenase activity in male C57BL/6 mice, RSC Adv. , 2017, 7, 56655.

130 Z. He, L. Lei, E. Kwek, Y. Zhao, J. Liu, W. Hao, H. Zhu, N. Liang, K. Y. Ma, H. M. Ho, W. He and Z. Y. Chen, Ginger attenuates trimethylamine-N-oxide (TMAO)-exacerbated disturbance in cholesterol metabolism and vascular inflammation, J. Funct. Foods, 2019, 52, 25-33.

131 Y. Hu, Y. Zhao, L. Yuan and X. Yang, Protective effects of tartary buckwheat flavonoids on high TMAO diet-induced vascular dysfunction and liver injury in mice, Food Funct., 2015, 6, 3359.

132 D. Ren, Y. Liu, Y. Zhao and X. Yang, Hepatotoxicity and endothelial dysfunction induced by high choline diet and the protective effects of phloretin in mice, Food Chem. Toxicol., 2016, 94, 203-212.

133 J. Guo, Y. Meng, Y. Zhao, Y. Hu, D. Ren and X. Yang, Myricetin derived from hovenia dulcis thunb. ameliorates vascular endothelial dysfunction and liver injury in high choline-fed mice, Food Funct., 2015, 6, 1620.

134 D. Li, D. Ren, Y. Lu and X. Yang, Protective effects of ursolic acid against hepatotoxicity and endothelial dysfunction in mice with chronic high choline diet consumption, Chem.-Biol. Interact., 2016, 258, 102107.

135 M. Jia, D. Ren, Y. Nie and X. Yang, Beneficial effects of apple peel polyphenols on vascular endothelial dysfunc- 
tion and liver injury in high choline-fed mice, Food Funct., 2017, 8, 1282.

136 C. Yang, Y. Zhao, D. Ren and X. Yang, Protective effect of saponins-enriched fraction of gynostemma pentaphyllum against high choline-induced vascular endothelial dysfunction and hepatic damage in mice, Biol. Pharm. Bull., 2020, 43, 463-473.

137 X. Wu, L. Chen, F. Zeb, Y. Huang, J. An, J. Ren, F. Yang and Q. Feng, Regulation of circadian rhythms by NEAT1 mediated TMAO-induced endothelial proliferation: A protective role of asparagus extract, Exp. Cell Res., 2019, 382, 111451.

138 M. Chen, B. Lu, Y. Li, Y. Wang, H. Zheng, D. Zhong, Z. Liao, M. Wang, F. Ma, Q. Liao and Z. Xie, Metabolomics insights into the modulatory effects of long-term compound polysaccharide intake in high-fat diet-induced obese rats, Nutr. Metab., 2018, 15, 8.

139 S. Liu, L. You, Y. Zhao and X. Chang, Wild Lonicera caerulea berry polyphenol extract reduces cholesterol accumulation and enhances antioxidant capacity in vitro and in vivo, Food Res. Int., 2018, 107, 73-83.

140 W. K. Wu, S. Panyod, C. T. Ho, C. H. Kuo, M. S. Wu and L. Y. Cheen, Dietary allicin reduces transformation of L-carnitine to TMAO through impact on gut microbiota, J. Funct. Foods, 2015, 15, 408-417.

141 M. L. Chen, L. Yi, Y. Zhang, X. Zhou, L. Ran, J. Yang, J. D. Zhu, Q. Y. Zhang and M. Mi, Resveratrol attenuates trimethylamine-N-oxide (TMAO)-induced atherosclerosis by regulating TMAO synthesis and bile acid metabolism via remodeling of the gut microbiota, mBio, 2016, 7, e02210-e02215.

142 Z. He, W. Hao, E. Kwek, L. Lei, J. Liu, H. Zhu, K. Y. Ma, Y. Zhao, H. M. Ho, W. S. He and Z. Y. Chen, Fish oil is more potent than flaxseed oil in modulating gut microbiota and reducing trimethylamine- N-oxide-exacerbated atherogenesis, J. Agric. Food Chem., 2019, 67, 1363513647.

143 Y. C. Koh, S. Li, P. Y. Chen, J. C. Wu, N. Kalyanam, C. T. Ho and M. H. Pan, Prevention of vascular inflammation by pterostilbene via trimethylamine-N-oxide reduction and mechanism of microbiota regulation, Food Funct., 2019, 63, 1900514.

144 P. Y. Chen, S. Li, Y. C. Koh, J. C. Wu, J. J. Yang, C. T. Ho and M. H. Pan, Oolong tea extract and citrus peel polymethoxyflavones reduce transformation of l-carnitine to trimethylamine-N-oxide and decrease vascular inflammation in l-carnitine feeding mice, J. Agric. Food Chem., 2019, 67, 7869-7879.

145 X. Zhang, Q. Wu, Y. Zhao and X. Yang, Decaisnea insignis seed oil inhibits trimethylamine-N-oxide formation and remodels intestinal microbiota to alleviate liver dysfunction in l-carnitine feeding mice, J. Agric. Food Chem., 2019, 67, 13082-13092.

146 T. Wu, Y. Gao, J. Hao, J. Geng, J. Zhang, J. Yin, R. Liu, W. Sui, L. Gong and M. Zhang, Capsanthin extract prevents obesity, reduces serum TMAO levels and modulates the gut microbiota composition in high-fat-diet induced obese C57BL/6J mice, Food Res. Int., 2020, 128, 108774.

147 T. Wu, Y. Gao, J. Hao, H. Yin, W. Li, J. Geng, R. Liu, W. Sui and M. Zhang, Lycopene, amaranth, and sorghum red pigments counteract obesity and modulate the gut microbiota in high-fat diet fed C57BL/6 mice, J. Funct. Foods, 2019, 60, 103437.

148 W. Cheng, J. Lu, B. Li, W. Lin, Z. Zhang, X. Wei, C. Sun, M. Chi, W. Bi, B. Yang, A. Jiang and J. Yuan, Effect of functional oligosaccharides and ordinary dietary fiber on intestinal microbiota diversity, Front. Microbiol., 2017, 8, 1750.

149 Q. Li, T. Wu, R. Liu, M. Zhang and R. Wang, Soluble dietary fiber reduces trimethylamine metabolism via gut microbiota and co-regulates host AMPK pathways, Mol. Nutr. Food Res., 2017, 61, 1700473.

150 Q. Li, H. Chen, M. Zhang, T. Wu and R. Liu, Altered short chain fatty acid profiles induced by dietary fiber intervention regulate AMPK levels and intestinal homeostasis, Food Funct., 2019, 10, 7174.

151 L. Li, B. Chen, R. Zhu, R. Li, Y. Tian, C. Liu, Q. Jia, L. Wang, J. Tang, D. Zhao, F. Mo, Y. Liu, Y. Li, A. N. Orkhov, D. Brömer, D. Zhang and S. Gao, Fructus Ligustri lucidi preserves bone quality through the regulation of gut microbiota diversity, oxidative stress, TMAO and Sirt6 levels in aging mice, Aging, 2019, 11, 9348-9368.

152 T. Yang, H. Qu, X. Song, Q. Liu, X. Yang, J. Xu, T. Yang, Z. Lan, W. Sha and H. Zhou, Luhong Granules prevent ventricular remodelling after myocardial infarction by reducing the metabolites TMAO and LPS of the intestinal flora, Evid. Based Complementary Altern. Med., 2019, 8937427.

153 J. Liu, T. Zhang, Y. Wang, C. Si, X. Wang, R. T. Wang and Z. Lv, Baicalin ameliorates neuropathology in repeated cerebral ischemia-reperfusion injury model mice by remodeling the gut microbiota, Aging, 2020, 12, 3792-3806.

154 P. G. Takis, M. E. Oraiopoulou, C. Konidaris and A. N. Troganis, (1)H-NMR Based Metabolomics study for the detection of the human urine metabolic profile effects of origanum dictamnus Tea ingestion, Food Funct., 2016, 7, 4104.

155 S. Dall'Acqua, M. Stocchero, I. Boschiero, M. Schiavon, S. Golob, J. Uddin, D. Voinovich, S. Mammi and E. Schievano, New findings on the in vivo antioxidant activity of Curcuma longa extract by an integrated (1) $\mathrm{H}$ NMR and HPLC-MS metabolomic approach, Fitoterapia, 2016, 109, 125-131.

156 P. Hernández-Alonso, D. Cañueto, S. Giardina, J. SalasSalvadó, N. Cañellas, X. Correig and M. Bulló, Effect of pistachio consumption on the modulation of urinary gut microbiota-related metabolites in prediabetic subjects, J. Nutr. Biochem., 2017, 45, 48-53.

157 E. Hill, H. Sapa, L. Negrea, K. Bame, T. Hostetter, H. Barkoukis, A. Dusso and M. Dobre, Effect of oat $\beta$-glucan supplementation on chronic kidney disease: A feasibility study, J. Renal Nutr., 2020, 30, 208-215. 
158 R. Obeid, H. M. Awwad, S. H. Kirsch, C. Waldura, W. Herrmann, S. Graeber and J. Geisel, Plasma trimethylamine-N-oxide following supplementation with vitamin D or D plus B vitamins, Mol. Nutr. Food Res., 2017, 61, 1600358.

159 G. Annunciata, M. Maisto, C. Schisano, R. Ciampaglia, V. Narciso, G. C. Tenore and E. Novellino, Effects of grape pomace polyphenolic extract (Taurisolo ${ }^{\circledR}$ ) in reducing TMAO serum levels in humans: preliminary results from a randomized, placebo-controlled, cross-over study, Nutrients, 2019, 11, 139.

160 G. Annunciata, M. Maisto, C. Schisano, R. Ciampaglia, V. Narciso, S. T. Hassan, G. C. Tenore and E. Novellino, Effect of grape pomace polyphenols with or without pectin on TMAO serum levels assessed by LC/MS-based assay: A preliminary clinical study on overweight/obese subjects, Front. Pharmacol., 2019, 10, 575.
161 G. C. Tenore, D. Caruso, G. Buonomo, M. D’Avino, R. Ciampaglia, M. Maisto, C. Schisano, B. Bocchino and E. Novellino, Lactofermented Annurca apple puree as a functional food indicated for the control of plasma lipid and oxidative amine levels: Results from a randomised clinical trial, Nutrients, 2019, 11, 122.

162 C. J. Angiletta, L. E. Griffin, C. N. Steele, D. J. Baer, J. A. Novotny, K. P. Davy and A. P. Neilson, Impact of short-term flavanol supplementation on fasting plasma trimethylamine $\mathrm{N}$-oxide concentrations in obese adults, Food Funct., 2018, 9, 5350.

163 M. E. Baugh, C. N. Steele, C. J. Angiletta, C. M. Mitchell, A. P. Neilson, B. M. Davy, M. W. Hulver and K. P. Davy, Inulin supplementation does not reduce plasma trimethylamine $\mathrm{N}$-Oxide concentrations in individuals at risk for Type 2 Diabetes, Nutrients, 2018, 10, 793. 